\title{
Recent insights from in vitro single-molecule studies into nucleosome structure and dynamics
}

\author{
Orkide Ordu $^{1}$ - Alexandra Lusser ${ }^{2}$ - Nynke H. Dekker ${ }^{1}$ \\ Received: 20 April 2016 / Accepted: 17 June 2016 / Published online: 18 October 2016 \\ (C) The Author(s) 2016. This article is published with open access at Springerlink.com
}

\begin{abstract}
Eukaryotic DNA is tightly packed into a hierarchically ordered structure called chromatin in order to fit into the micron-scaled nucleus. The basic unit of chromatin is the nucleosome, which consists of a short piece of DNA wrapped around a core of eight histone proteins. In addition to their role in packaging DNA, nucleosomes impact the regulation of essential nuclear processes such as replication, transcription, and repair by controlling the accessibility of DNA. Thus, knowledge of this fundamental DNA-protein complex is crucial for understanding the mechanisms of gene control. While structural and biochemical studies over the past few decades have provided key insights into both the molecular composition and functional aspects of nucleosomes, these approaches necessarily average over large populations and times. In contrast, single-molecule methods are capable of revealing features of subpopulations and dynamic changes in the structure or function of biomolecules, rendering them a powerful complementary tool for probing mechanistic aspects of DNA-protein interactions. In this review, we highlight how these singlemolecule approaches have recently yielded new insights into nucleosomal and subnucleosomal structures and dynamics.
\end{abstract}

This article is part of a Special Issue on 'DNA supercoiling, protein interactions and genetic function' edited by Laura Finzi and Wilma Olson

Nynke H. Dekker

N.H.Dekker@tudelft.nl

1 Bionanoscience Department, Kavli Institute of Nanoscience, Delft University of Technology, Van der Maasweg 9, 2629 HZ Delft, The Netherlands

2 Division of Molecular Biology, Biocenter, Medical University of Innsbruck, Innrain 80-82, 6020 Innsbruck, Austria
Keywords Single-molecule techniques $\cdot($ Sub)nucleosome structure and dynamics · Histones · Nucleosome remodeling · Post-translational modifications · Transcriptional barrier

\section{Introduction}

The genome of eukaryotic organisms ranges from millions to hundreds of billions of base pairs for different species and can be stretched accordingly to millimeters or meters (Kidwell 2002; Merhej and Raoult 2012; Zimmer 2007). These lengths are several orders of magnitude larger than the cell nucleus, with an average diameter of roughly $5 \mu \mathrm{m}$, and therefore the genome must be tightly packed in order to fit into the nucleus. To achieve an appropriate compaction level, eukaryotic organisms organize their genome in hierarchical protein-DNA assemblies termed chromatin that inevitably influence DNA accessibility during key cellular processes. Hence, revealing the details of chromatin structure is essential for understanding the regulation of the genome. A major milestone was set about forty years ago, when chromatin was first reported to comprise a repeating unit of $\sim 200 \mathrm{bp}$ of DNA wrapped around a core of histone proteins (Kornberg 1974; Olins and Olins 1974; Oudet et al. 1975). This basic component of chromatin, termed the nucleosome, has been a central subject of research ever since.

Bulk studies based on traditional biochemical approaches from molecular and structural biology have provided invaluable insight into nucleosome structure and function (Kornberg 1977; Kornberg and Lorch 1999; Li et al. 2007; McGhee and Felsenfeld 1980; Workman and Kingston 1998; Zentner and Henikoff 2013). However, these methods only reflect collective properties of samples due to ensemble and time-averaging. Partial features, such as rare or transient events, and especially 
the intrinsic dynamics of usually heterogeneous biological samples cannot be resolved.

When many identical biomolecules are present in a solution, each one can occupy any one of a number of different conformational states with similar energies. Assuming for simplicity that each state corresponds to a distinct, visible color, the whole solution will at any given time display a color that reflects the average of all the individual molecules, which will each occupy different states with their corresponding distinct colors. Likewise, the observation of a single molecule on a long timescale, during which the molecule can convert between all states with the corresponding colors, will also yield an average color. The heterogeneity arising from both static differences between single biomolecules and individual dynamic changes in, for example, their structure, function or chemical modification remains therefore hidden.

Details of molecular properties can be revealed by directly studying single molecules over time. However, the insights that can be obtained here strongly depend on the characteristics of the experimental system in use and the spatial and temporal resolution of the applied technique. During the observation within a certain time frame, the molecule might convert between barely different states at such high speed that intermediates would be averaged out and obscured. Hence, since the 1990s the development of single-molecule studies has not only included the interrogation of different biological systems but also the continued improvement of their accuracy and sensitivity (Dulin et al. 2013; Eigen and Rigler 1994; Hell 2009; Huang et al. 2009; Jares-Erijman and Jovin 2003; Joo et al. 2008; Moerner 2007; Neuman and Nagy 2008; Tinoco and Gonzalez 2011; Weiss 1999). Single-molecule techniques have become a highly suitable tool for investigating DNAprotein interactions at the molecular level (Duzdevich et al. 2014; Heller et al. 2014; Monico et al. 2013). In the context of DNA compaction into chromatin, they have also yielded new, complementary findings on the structure, function and especially dynamics of chromatin and nucleosomes (Buning and van Noort 2010; Choy and Lee 2012; Killian et al. 2012; Lavelle et al. 2011; Petesch and Lis 2012; Zlatanova and Leuba 2003).

In this review, we will introduce the most commonly used single-molecule techniques in nucleosome research and present the recent insights they have provided into nucleosomal and subnucleosomal structure, function and dynamics within the last 3 years.

\section{Single-molecule techniques in nucleosome research}

Over the past two decades, time-resolved observation and manipulation of single molecules have become very powerful means to investigate biological systems. The major singlemolecule techniques enable the researchers to either directly visualize or influence individual molecules to reveal molecular details of their structure, function and dynamics on the nanometer scale. Substantial technical advances in optical microscopy and fluorescent probes have made fluorescence microscopy and fluorescence spectroscopy routine methods for directly visualizing and observing single molecules over time (Giepmans et al. 2006; Moerner and Fromm 2003). The manipulation of individual molecules using force spectroscopy has become possible by the development of trapping methods with different types of force transducers (Neuman et al. 2007). Among these techniques, atomic force microscopy (AFM) is a unique method that enables either the direct observation or the manipulation of single molecules by imaging or trapping, respectively (Butt et al. 2005; Hansma and Hoh 1994). The most commonly employed single-molecule techniques and their associated specific experimental approaches are described in the following subsections.

\section{Fluorescence microscopy and fluorescence spectroscopy}

Fluorescence microscopy essentially relies on the detection of light emitted at a specific wavelength by specific molecules (fluorophores) that are fused to the biomolecule of interest following their excitation at an initial, typically shorter, wavelength. The type and precise characteristics of these fluorophores will ultimately determine the efficiency and applicability of this technique to a specific study (Berlier et al. 2003; Shaner et al. 2005). Fluorescent samples can be excited either in a large or a small area depending on the design of the microscope. In wide-field microscopy, the sample is illuminated by a nearly collimated light beam, resulting in the simultaneous excitation of numerous fluorophores at different depths of focus and therefore in considerable background noise (Funatsu et al. 1995; Schmidt et al. 1996). This out-offocus fluorescence is substantially decreased in total internal reflection fluorescence microscopy (TIRFM), which restricts the illumination depth to about $100 \mathrm{~nm}$ via a highly localized, quickly decaying electromagnetic field (evanescent field) that is generated at the sample surface (Axelrod 1981, 2001). Confocal microscopy reduces the excitation volume and almost entirely eliminates out-of-focus light by using a focused laser beam and a spatial pinhole positioned just before the detector (Minsky 1988; Nie et al. 1994). These excitation methods are used in combination with different fluorescence spectroscopy techniques depending on the research question.

Förster or fluorescence resonance energy transfer (FRET) is a well-established method to study the structural dynamics of single molecules. It is based on the non-radiative energy transfer between two fluorescent probes in very close proximity (Förster 1948; Ha et al. 1996). An excited fluorophore (donor) can non-radiatively transfer its energy to another, sufficiently close fluorophore (acceptor) which then emits fluorescent light (Fig. 1a). By monitoring this energy transfer and 

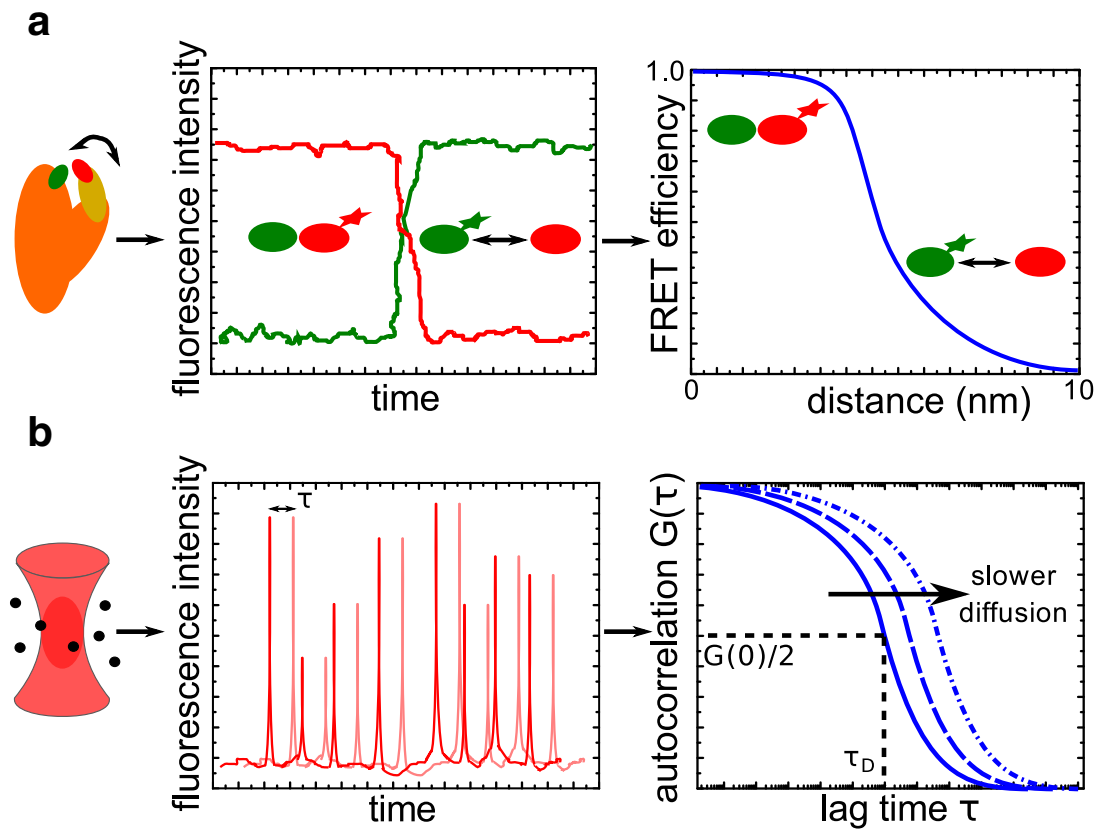

Fig. 1 Sketched examples of fluorescence spectroscopy techniques. a Single-molecule fluorescence resonance energy transfer (smFRET). A dynamic molecule (orangelyellow) is labelled with a donor (green) and an acceptor $(r e d)$ fluorophore. When the fluorophores are close together within $10 \mathrm{~nm}$, the excited donor will transfer its energy to the acceptor for fluorescence emission. For distances of $>10 \mathrm{~nm}$, only the donor will fluoresce. The recorded time-resolved fluorescence signals of the donor (green) and acceptor (red) are used to calculate the efficiency of FRET. b

its efficiency in real-time, the dynamics and distances of interand intramolecular interactions involved in conformational changes can be revealed on the scale of $1-10 \mathrm{~nm}$. This method was first applied to nucleosomes for studying their structural dynamics (Tomschik et al. 2005). The efficiency of FRET between a donor and an acceptor fluorophore each located on the nucleosomal DNA at $\sim 45$ bp from its entry and exit sites showed fast dynamic changes in nucleosome structure between a long-lived, fully wrapped state $(2-5 \mathrm{~s})$ and a transient, substantially unwrapped conformation (100-200 $\mathrm{ms})$. This work represents the first attempt to directly investigate the dynamic nature of nucleosomes, and the results suggest a potential mechanism by which DNA accessibility for DNA-binding proteins can be regulated. However, the observed dynamics was highly affected by fluorophore blinking, which influenced the results and was corrected for in a later publication by the authors (Tomschik et al. 2009). Different FRET-based assays have subsequently been developed and used, enabling more accurate, robust and reliable insights into nucleosome structure and dynamics (Blosser et al. 2009; Koopmans et al. 2007; North et al. 2012; Simon et al. 2011).

Another frequently used single-molecule fluorescence technique is fluorescence correlation spectroscopy (FCS), which allows the study of the dynamics of individual molecules as they freely diffuse in solution (Elson and Magde
Fluorescence correlation spectroscopy. Very few molecules (black) diffusing through the tiny excitation volume generated in a confocal microscope are excited for fluorescence emission. The time-resolved fluorescence signal is recorded and analyzed by autocorrelation, i.e. checking its similarity to its replicas shifted by different lag times $(\tau)$. The resulting autocorrelation function $[G(\tau)]$ yields the characteristic parameters of the underlying process, such as the diffusion time $\left(\tau_{D}\right)$ at about half of the amplitude $[G(0) / 2]$

1974; Magde et al. 1972, 1974; Rigler et al. 1993). FCS is based on the correlation analysis of fluctuations in the timeresolved fluorescence signal that arises from very few molecules diffusing through the tiny excitation volume $\left(\sim 10^{-3}\right.$ pl) generated in a confocal microscope (Fig. 1b). Essentially, the fluorescence signal is compared to its replicas calculated at different lag times to check their similarity and reveal repetitive patterns due to the underlying physical processes, such as free diffusion, chemical reaction or conformational changes. The resulting mathematical expression (the autocorrelation function) yields the characteristic parameters of these processes, such as diffusion constants, concentrations, hydrodynamic radii or reaction rates. FCS was first used to investigate the structural dynamics of nucleosomes in combination with FRET ( $\mathrm{Li}$ et al. 2005). The un- and rewrapping rates of the nucleosome were initially determined indirectly via FRET by trapping the open conformation using a site-specific DNA-binding protein. FCS measurements were then performed on nucleosomes labeled with either donor only or a donor-acceptor pair to directly observe conformational changes for a more reliable interpretation of the kinetics. The results obtained from both approaches led to the conclusion that nucleosomal DNA unwraps on a timescale of $250 \mathrm{~ms}$ and rewraps more rapidly within 10-50 ms. Subsequent efforts using this technique have provided additional insights into nucleosome structure 
and dynamics (Bohm et al. 2011; Gansen et al. 2009; Koopmans et al. 2009; Poirier et al. 2009; Tims et al. 2011).

These studies convincingly demonstrate the great power of the most commonly used single-molecule fluorescence techniques to visualize DNA-protein interactions. When used in combination, they can yield complementary insights that allow the researchers to draw more reliable conclusions. However, the data acquisition, analysis and interpretation in fluorescence microscopy studies must always take into account the many factors related to the photophysics of the fluorophores (Ha and Tinnefeld 2012). This issue is entirely eliminated in force spectroscopy techniques which rely on the detection of light scattered by micron-sized massive particles. The most common approaches of these manipulation methods are presented below.

\section{Force spectroscopy}

The basis of force spectroscopy techniques is the specific attachment of single molecules between a substrate and a force transducer by interacting proteins or organic or engineered compounds. This tethering enables manipulation of the molecules by the application of forces and, in some cases, torques. The readouts provided by the force transducers are tracked in real-time, allowing for the investigation of primarily mechanical properties of the sample. Depending on the biological question of interest, different force transducers are used together with distinct methods for trapping and monitoring them.

In optical tweezers (OT) dielectric micron-sized particles are captured in the focus of an intense laser beam exerting a force due to the light gradient (Ashkin 1992; Ashkin et al. 1986; Smith et al. 1996). In biological applications, OT typically involve a nucleic acid molecule tethered between an optically trapped bead and a substrate, which can either be the surface of the sample holder or another, fixed bead held by a micropipette or even a second optical trap (Fig. 2a). The underlying mechanism is based on controlling the position of the trapped bead and, thereby, the molecule's extension. Therefore, this type of trapping is referred to as an extension clamp. Moving the focused laser beam with the trapped bead allows the manipulation of the molecule by inducing a concomitant change in its extension, which concurrently affects the molecule's tension that is related to the applied forces ranging between 0.1 and $100 \mathrm{pN}$. The bead's position is recorded indirectly by detecting the laser signal on a position-sensitive device, which enables the simultaneous measurement of force and extension, the two key quantities of force spectroscopy. This method was first used in chromatin research to study the structure of native chromatin fibers extracted from chicken erythrocytes (Cui and Bustamante 2000). The mechanical stretch-release manipulation revealed a reversible decondensation of the fibers at low forces $(<6 \mathrm{pN})$, which was attributed to internucleosomal interactions. Specifically, the fibers showed a pronounced transition between condensation and decondensation at 5-6 pN under physiological salt concentrations, indicating strong internucleosomal interactions with energies comparable to the thermal energy. Upon pulling at high forces $(>20 \mathrm{pN})$, the fibers were observed to undergo irreversible changes in their extension, which was explained by the possible eviction of the histone proteins during this mechanical unfolding. The results of this study led to the first insights into the energy landscape of chromatin structure and also suggested a considerable dynamic nature due to thermal fluctuations. It was followed by many other OT-based assays that shed more light on the structure and dynamics of nucleosomes (Bennink et al. 2001; Bintu et al. 2012; Brower-Toland et al. 2005; Gemmen et al. 2005; Hall et al. 2009; Hodges et al. 2009; Jin et al. 2010; Mack et al. 2012; Pope et al. 2005; Shundrovsky et al. 2006; Sirinakis et al. 2011; Zhang et al. 2006).

Another very common technique used for mechanical manipulation of single molecules is magnetic tweezers (MT). In this method, magnetic beads are trapped by permanent or electrical magnets that exert a force as a result of a magnetic field gradient (Amblard et al. 1996; Crick and Hughes 1950; Smith et al. 1992; Strick et al. 1996, 1998; Ziemann et al. 1994). In the most common designs a nucleic acid molecule is tethered between a magnetic bead and the surface of the sample holder at its two extremities and manipulated using permanent magnets (Fig. 2b). Vertical movement of the magnets results in a corresponding change of the applied force, ranging from $10^{-3}$ to $100 \mathrm{pN}$, and a concomitant change of the bead's position, which is directly recorded by video microscopy with a charge-coupled-device (CCD) camera. As the applied force is the parameter that is precisely controlled in this technique, the underlying mechanism is also referred to as force clamp. However, besides forces, MT can also apply torques by rotating the magnets. MT were first used in chromatin research to study the time- and force-dependence of the assembly and disassembly of chromatin fibers (Leuba et al. 2003). Fibers were found to assemble only at forces up to 10 $\mathrm{pN}$, while assemblies at the higher forces within this range were observed to be reversible. These results revealed a strong dependency of chromatin assembly on the force applied to the DNA and illustrated the dynamic equilibrium of this process. Translated to a possible scenario in the cell, these experiments allow conclusions to be drawn on the potential fate of chromatin/nucleosomes under forces exerted by enzymes during DNA-templated processes. For example, the forces generated by the $E$. coli RNA polymerase (RNAP) were shown to be in the picoNewton range (Wang et al. 1998). Subsequent MT studies confirmed and further refined these results to a more detailed picture of the structure and dynamics of chromatin and nucleosomes (Kruithof et al. 2009; Lia et al. 2006; Simon et al. 2011; Vlijm et al. 2012; Yan et al. 2007). The 
a

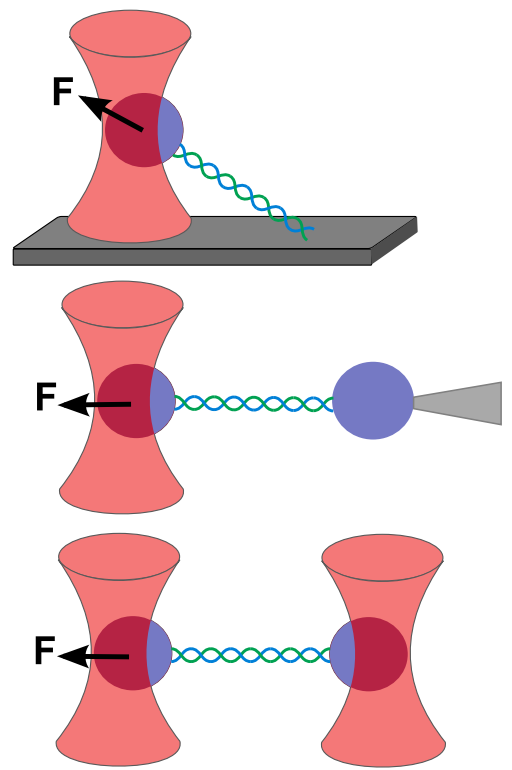

Fig. 2 Overview of force spectroscopy techniques. a Optical tweezers. A DNA molecule (blue/green) is tethered between an optically trapped dielectric microsphere (violet) and either the glass coverslip (top) or another bead fixed using a micropipette (middle) or a second optical trap (bottom). Moving the optical trap will change the tether's extension and tension related to the applied forces $(F)$, ranging between 0.1 and 100 pN (extension clamp). b Magnetic tweezers. A DNA molecule is tethered between the glass coverslip and a magnetic bead that is trapped using a

specific function of the nucleosome in higher-order folding of chromatin based on inter-nucleosomal interactions has also been assessed using single-molecule force and torque spectroscopy, but it still remains elusive due to additional restrictions on the electrostatics, topology and elasticity of the complex (Chien and van Noort 2009; Lavelle et al. 2010). While MT and OT have become the routine approaches for force spectroscopy due to their simple yet robust principles, they are, however, limited to mechanical manipulation of samples and do not allow direct observation.

\section{Atomic force microscopy}

Atomic force microscropy (AFM), also called scanning force microscopy (SFM), is a technique that is capable of either observing or manipulating single molecules on the same instrument by imaging or force spectroscopy, respectively (Binnig et al. 1986; Florin et al. 1994). Both principles are based on the use of a cantilever as the force transducer. This cantilever is either scanned over a sample to obtain a topographical image by means of atomic interactions or tethered to one extremity of an individual molecule for its mechanical manipulation. Its interaction with the sample involving forces ranging between 10 and $10^{4} \mathrm{pN}$ leads to the bending of the cantilever, which is tracked by the use of a laser beam directed on the cantilever and b

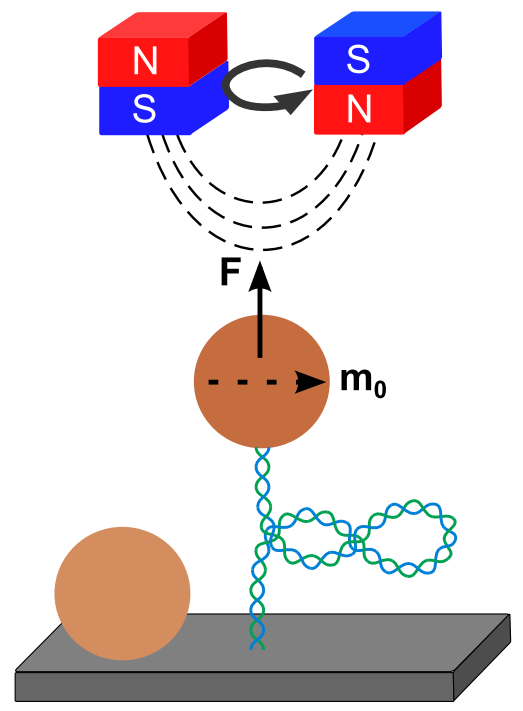

pair of cubic permanent magnets (red/blue) which accurately exert forces ranging between $10^{-3}$ and $100 \mathrm{pN}$ due to the magnetic field gradient (force clamp). Due to an induced horizontal magnetic moment $\left(m_{0}\right)$, the bead is also torsionally trapped, which allows the application of torques by rotating the permanent magnets. Torque application leads to supercoiling of the DNA molecule and the formation of plectonemes (circles of DNA). Non-magnetic reference beads adhered to the surface are used to correct for drift

reflected onto a position-sensitive device (Fig. 3). In this way, either the topology of a sample or the extension of a molecule can be indirectly read out with near atomic resolution $(\leq 1 \mathrm{~nm})$ by controlling the position of the cantilever. Hence, like OT, AFM also operates primarily as an extension clamp in force spectroscopy. In nucleosome research, this technique is mainly used for imaging, as the applied forces are in the higher range of the molecular scale and the distinct structures of chromatin, such as mono- and polynucleosomes, or higher-order foldings into fibers are very suitable to study using this specific approach. The first AFM study was performed on nucleosome arrays in order to directly observe and characterize their structural details (Allen et al. 1993). This work convincingly illustrated the applicability of AFM imaging for highresolution studies on nucleosome structure and was followed up by many researchers investigating the dynamics, as well as the role of nucleosomes in DNA accessibility (Bintu et al. 2011; Dalal et al. 2007; Dimitriadis et al. 2010; Miyagi et al. 2011; Shlyakhtenko et al. 2009; Yoda et al. 2000). Therefore, AFM represents another widely used technique in nucleosome research in addition to fluorescence and force spectroscopy. In the following sections we highlight recent insights into (sub)nucleosomal structure and dynamics from studies using most of the specific single-molecule approaches presented here. 
Fig. 3 Principles of atomic force microscopy. A cantilever (orange) is used to exert atomic forces on the sample. Their interaction leads to distortions of the cantilever which is recorded using a laser beam (red) that is reflected onto a position-sensitive device such as a quadrant photodiode (blue). a The cantilever can scan the sample to obtain a topographical image. b A DNA molecule is tethered between the glass coverslip and the cantilever to exert forces between 10 and $10^{4} \mathrm{pN}$ for force spectroscopy



\section{Nucleosome structure and dynamics}

The nucleosome consists of $147 \mathrm{bp}$ of DNA wrapped $\sim 1.7$ times in a left-handed superhelix around a discoidal protein structure of $\sim 5 \mathrm{~nm}$ in height and $\sim 7 \mathrm{~nm}$ in diameter formed by eight histones (Davey et al. 2002; Luger et al. 1997; Richmond et al. 1984) (Fig. 4). This histone octamer contains two copies of each of the so-called core histones $\mathrm{H} 2 \mathrm{~A}, \mathrm{H} 2 \mathrm{~B}, \mathrm{H} 3$ and $\mathrm{H} 4$ that are assembled into four heterodimers, i.e. two $\mathrm{H} 2 \mathrm{~A} / \mathrm{H} 2 \mathrm{~B}$ and two $\mathrm{H} 3-\mathrm{H} 4$ dimers, by short-range interactions between the central $\alpha$-helical histone-fold domains in a 'handshake' manner (Arents et al. 1991; Klug et al. 1980). The two H3-H4 dimers join to form a tetramer through the four-helix bundles of the $\mathrm{H} 3$ histones centered on the pseudotwofold symmetry (dyad) axis, while the two H2A/H2B dimers attach to the tetramer via similar four-helix bundle interactions between the $\mathrm{H} 2 \mathrm{~B}$ and $\mathrm{H} 4$ histones. Each core histone further features a flexible N-terminal tail, while the histone $\mathrm{H} 2 \mathrm{~A}$ additionally exhibits a $\mathrm{C}$ terminal tail. All of the histones are highly positively charged, and as such they balance the negative charge of the DNA. Hence, the histone octamer is only found to be stable in the presence of DNA or at high salt concentrations $(\sim 2 \mathrm{M})$, and it dissociates into the (H3$\mathrm{H} 4)_{2}$ tetramer and the two $\mathrm{H} 2 \mathrm{~A} / \mathrm{H} 2 \mathrm{~B}$ dimers at physiological conditions (Eickbush and Moudrianakis 1978). Likewise, the nucleosome is assembled in a stepwise manner by the initial binding of the $(\mathrm{H} 3-\mathrm{H} 4)_{2}$ tetramer to the DNA and the subsequent incorporation of the H2A/H2B dimers (Polo and Almouzni 2006). However, the nucleosome complex resulting from this well-defined assembly pathway is not static, but a highly dynamic entity. Its inter-dependent structural, mechanical, chemical and functional properties are continuously altered by different mechanisms, such as intrinsic dynamics, chemical modifications of the DNA and histones, ATPdependent remodeling, as well as by forces and torques exerted by genome processing enzymes. The concerted action of all of these mechanisms makes it very difficult to study this complex system as a whole using singlemolecule techniques. Such methods can, however,
Fig. 4 Structure of the nucleosome. A total of $147 \mathrm{bp}$ of DNA (grey) are wrapped around a discoidal protein structure containing two copies of the eight core histones $\mathrm{H} 2 \mathrm{~A}$ (magenta), $\mathrm{H} 2 \mathrm{~B}$ (orange), $\mathrm{H} 3$ (green) and $\mathrm{H} 4$ (blue) in a left-handed superhelix. a Top view. b Side view along the pseudo-twofold symmetry (dyad) axis. The images were created from the structural data in the RCSB Protein Data Bank (PDB) with the identification code $1 \mathrm{AOI}$ (Luger et al. 1997) using the PyMOL Molecular Graphics System, Version 1.7.2.1 Schrödinger, LLC

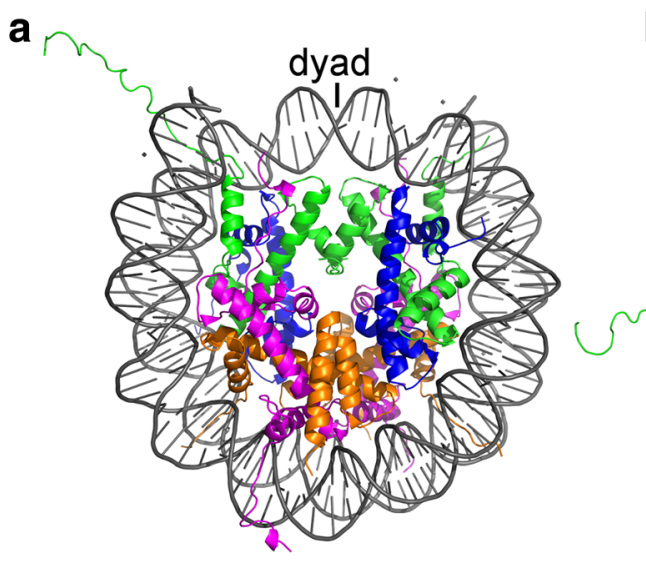

b

b

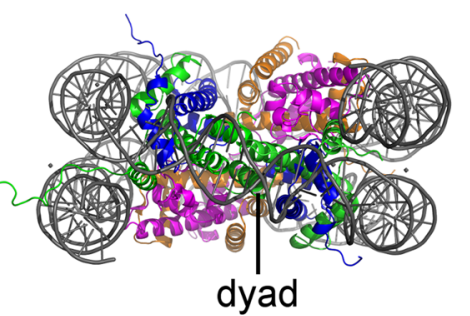


provide invaluable insights into the different individual mechanisms and their impact on nucleosome structure, dynamics and function (Choy and Lee 2012; Killian et al. 2012).

\section{Intrinsic nucleosome dynamics}

The observation of nucleosomes at the single-molecule level have revealed that their structure is intrinsically dynamic. As mentioned above, about $30 \mathrm{bp}$ at the entry and exit sites of the nucleosomal DNA were reported to spontaneously unwrap and rewrap on a timescale between 10-300 ms (Koopmans et al. 2007; Li et al. 2005; Miyagi et al. 2011) (Fig. 5a). However, until recently this phenomenon of DNA breathing was studied either indirectly with assays using DNA-binding proteins to trap the open nucleosome conformation or directly using methods limited in their specific time resolution for technical reasons. Very recently, the transient unwrapping and rewrapping of nucleosomal DNA ends has been identified on a timescale of 1$10 \mathrm{~ms}$ using a novel single-molecule technique combining single-molecule FRET (smFRET) and FCS with stochastic data analysis based on maximum likelihood estimation (MLE) (Wei et al. 2015). This approach enables the study of the structural dynamics of biomolecules on the sub-microsecond timescale considering the photophysical properties of the fluorophores. By this means, this study provided the first direct evidence that DNA breathing is a very fast process. In another recent work, the first experimental evidence for a novel spontaneous transition of nucleosome structure, called gaping, was reported using smFRET (Ngo and Ha 2015). This phenomenon refers to the transient opening of the two turns of nucleosomal DNA with respect to each other along the superhelical axis (Fig. 5b). Different labeling schemes were used to study this conformational change associated with an estimated distance change of $0.5-1 \mathrm{~nm}$ and a timescale of 1-10 $\mathrm{min}$ (Fig. 5c, d). However, due to technical limitations in terms of resolution owing to the use of FRET and the labeling strategy based on the use of a linker, further high-resolution studies are needed to reveal the details of this phenomenon, including potential structural changes in the histone octamer. Further evidence supporting these recent findings will certainly have strong implications for the role of the intrinsic structural dynamics of nucleosomes as a major mechanism for regulating DNA accessibility in the context of genomic processes.

\section{The sequence of the nucleosomal DNA}

The structural, mechanical and functional properties of nucleosomes have also been suggested to depend on the underlying DNA sequence due to the influence of the latter on nucleosome positioning (Widom 2001). This notion has provoked high interest in investigating nucleosomal DNA sequences to identify weak and strong nucleosomes and has led to the development of different artificial sequences which have become widely used in in vitro studies (Trifonov and Nibhani 2015). Recently, the influence of DNA sequence on nucleosome structure was studied using a single-molecule approach

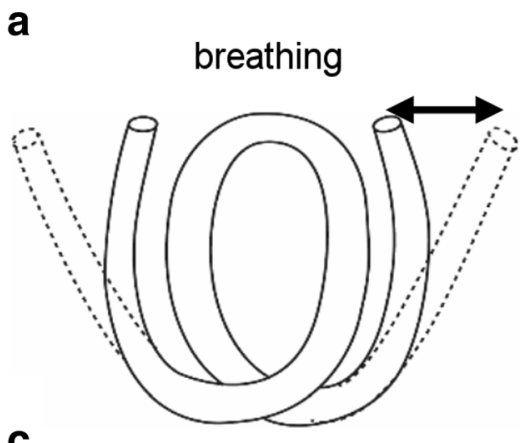

C

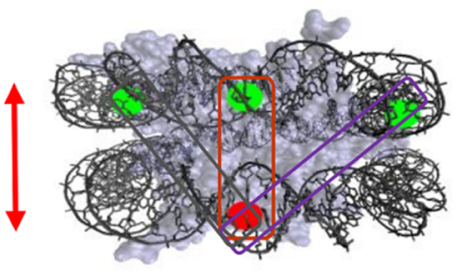

b

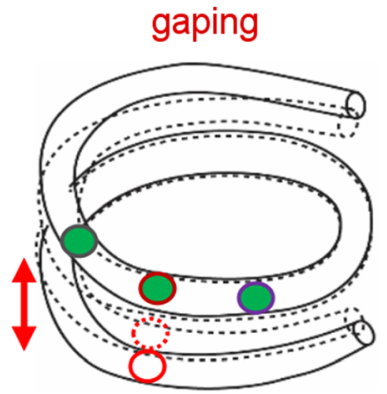

d



Fig. 5 Intrinsic nucleosome dynamics. a The nucleosomal DNA ends transiently wrap and unwrap from the histone octamer (breathing), indicated by the black arrow. b The two turns of nucleosomal DNA transiently open with respect to each other along the superhelical axis (gaping), depicted by the red arrow. c Different labelling schemes to identify the gaping transition which is best characterized by the FRET pair encircled in red. $\mathbf{d}$ Time-resolved fluorescence signals of the donor

(green) and acceptor (red) fluorophore from the best characterized FRET pair are recorded and yield the FRET efficiency, showing a slight increase over several minutes due to gaping. All panels (a-d) are figures reprinted with minor changes from Ngo and Ha (2015), Copyright (2015), used under a CC BY 4.0 license (http://creativecommons.org/licenses/by/4.0/). This figure is not included in the present article's Creative Commons license 
combining smFRET and OT (Ngo et al. 2015). This assay allowed the simultaneous manipulation and observation of the nucleosome to probe force-induced local conformational changes. Nucleosomes were found to disassemble by asymmetric and directional unwrapping under force, whereby the relative stiffness of different regions of the nucleosomal DNA dictated the unwrapping direction, with a preference for starting from the stiffer side. When the DNA exhibited similar flexibility on both sides, nucleosomes unwrapped stochastically from either side. Both ends further showed an interplay in which the opening of one end stabilized the other, indicating that even small differences in DNA flexibility on either side could lead to an asymmetric stability of the nucleosome. These findings clearly demonstrate the influence of local DNA flexibility, caused by its sequence composition, on nucleosome stability and DNA accessibility. In a more general context, they suggest a new mechanism contributing to the regulation of DNA accessibility by the nucleosomal DNA sequence and its modifications. However, if and to what extent sequence-dependent effects on nucleosomal DNA dynamics play a role for in vivo processes awaits future investigation, which will likely be challenging, as many different mechanisms cooperate to influence nucleosome (re)organization in the cell.

\section{Post-translational modification of histones}

One well-established mechanism that substantially influences nucleosome structure and dynamics is the post-translational modification (PTM) of the unstructured histone tails protruding from the nucleosome core complex at specific positions (Bowman and Poirier 2015). This form of dynamic chemical alteration of the histones mediated by a great number of dedicated enzymes has been shown to change the structural and dynamic properties of nucleosomes by affecting histoneDNA or histone-histone interactions. The best-studied chemical modifications include histone acetylation and phosphorylation. Their effects on nucleosome structural dynamics were studied recently by complementing different biochemical assays with smFRET (Brehove et al. 2015). Phosphorylation of tyrosine 41 and threonine 45 of histone $\mathrm{H} 3$ located in the nucleosomal core near the DNA entry-exit sites was found to enhance DNA accessibility by threefold, as did histone acetylation of lysine 56 in the same region. Remarkably, simultaneous phosphorylation and acetylation were observed to increase DNA accessibility by an order of magnitude. Although DNA accessibility was tested indirectly by a protein-binding assay, which does not allow direct quantification of the intrinsic dynamics of nucleosomes, the study still clearly demonstrates the significant effect of PTMs on DNA unwrapping dynamics. In a broader context, these results suggest that particularly PTMs of the globular domains of the histones have the ability to directly affect nucleosome stability by impacting histone-DNA interactions while modulating the ability of the nucleosome to bind regulatory factors. A large and increasing number of identified PTMs awaits further study at the single-molecule level to advance our understanding of nucleosome structure and dynamics (Arnaudo and Garcia 2013).

\section{ATP-dependent remodeling}

Complementary to the chemical modification of histones, another mechanism that affects the stability and dynamics of nucleosomes is mediated by enzymes that actively reorganize nucleosome structure. These ATP-dependent chromatin remodelers catalyze changes in nucleosome position and composition by inducing nucleosome sliding or (partial) disassembly/assembly of histones upon ATP-hydrolysis (Clapier and Cairns 2009). The nucleosome remodeling process by the ATP-dependent chromatin assembly and remodeling factor (ACF) that contains a catalytic subunit belonging to the imitation switch (ISWI)-family and generates uniformly spaced nucleosomal arrays was recently studied in molecular detail using smFRET (Hwang et al. 2014). This approach allowed for the time-dependent observation of DNA translocation upon remodeling by ACF. Both the linker DNA and the histone $\mathrm{H} 4$ tail were found to affect DNA translocation by increasing the pause durations in the remodeling process. The catalytic and accessory subunit of ACF, Snfh2 and Acf1, respectively, were observed to cooperate in detecting the linker DNA with the help of the histone H4 tail. For short linker DNA lengths, Acfl preferably bound to the N-terminal region of the histone $\mathrm{H} 4$ tail, which resulted in autoinhibition of the ATPase activity of Snfh2 while possibly increasing pause durations. With increasing linker DNA lengths, however, Acfl changed its binding preference towards the linker DNA by releasing the histone $\mathrm{H} 4$ tail, which in turn relieved the autorepression mechanism and resulted in activation of the Snf2h ATPase. These results indicate that DNA linker length and the histone $\mathrm{H} 4$ tail are important components of nucleosome remodeling by enzymes of the ISWI-family and suggest a potential regulatory mechanism to direct nucleosome spacing. Many chromatin remodelers of different families have been identified, and the details of their remodeling mechanism still need to be studied (Hota and Bartholomew 2011). As demonstrated by the results of this study, single-molecule techniques are highly suitable for this purpose. The bigger challenge seems to be the investigation of all mechanisms affecting nucleosome structure and dynamics in combination.

\section{Genome processing enzymes}

In addition to the mechanisms presented above that specifically target nucleosomes, non-specific external events 
mediated by other factors influence nucleosome structure and dynamics. Certain enzymes have to exert forces and torques in order to perform their tasks in processing the genome. In a recent study, the effect of force and torque on nucleosome structure was investigated using an angular optical trapping method called the optical torque wrench (OTW) (Sheinin et al. 2013). In addition to allowing the manipulation of biomolecules by means of force, this OTbased technique allows the application and measurement of torque (La Porta and Wang 2004). Qualitatively, torque was found to only have a modest effect on nucleosome disassembly. The unwrapping of nucleosomes always followed a distinct two-step pattern, namely, a sudden release of nucleosomal DNA at lower forces $(<6 \mathrm{pN})$, attributed to the outer turns around the $\mathrm{H} 2 \mathrm{~A} / \mathrm{H} 2 \mathrm{~B}$ dimers, and another release at higher forces $(\geq 6 \mathrm{pN})$, assigned to the inner turn around the $(\mathrm{H} 3-\mathrm{H} 4)_{2}$ tetramer. This interpretation and the details of nucleosome unwrapping, however, might need to be reconsidered after the recent observation of asymmetric unwrapping reported above. Quantitatively, however, torque was observed to significantly affect the disruption forces by stabilizing the outer turns and destabilizing the inner turn. Remarkably, the application of positive torque additionally led to a striking loss of $\mathrm{H} 2 \mathrm{~A} / \mathrm{H} 2 \mathrm{~B}$ dimers, whereas the $(\mathrm{H} 3-$ $\mathrm{H} 4)_{2}$ tetramer remains stably bound to the DNA. These findings suggest a potential role of torque and supercoiling in regulating DNA-templated processes by facilitating the removal of the $\mathrm{H} 2 \mathrm{~A} / \mathrm{H} 2 \mathrm{~B}$ dimers. More recently, the effect of supercoiling on nucleosome structure was investigated using AFM and FCS (Elbel and Langowski 2015). The architecture of the nucleosomes was revealed by AFM imaging, while their stability was studied by measuring the diffusion constants upon salt-induced destabilization using FCS. Nucleosome structure was found to be dependent on the sign and density of the superhelical turns. Negative supercoiling resulted in more compact and stable nucleosomes that were resistant to changes in salt concentration. In contrast, nucleosomes reconstituted on either relaxed or positively supercoiled DNA were observed to be more open and prone to salt-induced disassembly. Destabilization of these nucleosomes, leading to the enhanced eviction of the $\mathrm{H} 2 \mathrm{~A} / \mathrm{H} 2 \mathrm{~B}$ dimers, was observed to start at $\sim 600-800 \mathrm{mM}$ monovalent salt concentration. The $(\mathrm{H} 3-\mathrm{H} 4)_{2}$ tetramer, however, was found to dissociate later at salt concentrations of $>1000 \mathrm{mM}$. These results from the combined approach of imaging and fluorescence spectroscopy clearly demonstrate the significant impact of DNA topology on nucleosome structure and stability and further support the notion of DNA supercoiling as a potential mechanism for regulating the genome by facilitating histone eviction. Investigating structural transitions in the histone octamer itself under torque appears to be a logical next step to reveal more details of the force- and torque-induced disassembly of nucleosomes. The single-molecule approaches, however, need to take into account the biological relevance of all components, such as the range of the applied forces and torques, as well as the salt concentrations used. The regular observation of distinct behavior for the $\mathrm{H} 2 \mathrm{~A} / \mathrm{H} 2 \mathrm{~B}$ dimers and the $(\mathrm{H} 3-\mathrm{H} 4)_{2}$ tetramer raises more questions about the architecture and dynamics of subnucleosomal structures which will be discussed in the next section.

\section{Subnucleosomal structures and dynamics}

As mentioned above, nucleosomes have been observed to lose their outer $\mathrm{H} 2 \mathrm{~A} / \mathrm{H} 2 \mathrm{~B}$ dimers under force, torque or changes in salt concentration, while $(\mathrm{H} 3-\mathrm{H} 4)_{2}$ tetramers remain bound to the DNA. These findings indicate the existence of intermediate nucleosome states, several of which have in fact been reported in different studies (Andrews and Luger 2011; Lavelle and Prunell 2007; Luger et al. 2012; Zlatanova et al. 2009). The assembly of nucleosomes happens in a stepwise manner through the initial binding of the $(\mathrm{H} 3-\mathrm{H} 4)_{2}$ tetramer to the DNA, followed by the incorporation of the two H2A/H2B dimers (Polo and Almouzni 2006). In the absence of DNA but under otherwise physiological conditions, the histone octamer itself dissociates into the $(\mathrm{H} 3-\mathrm{H} 4)_{2}$ tetramer and $\mathrm{H} 2 \mathrm{~A} / \mathrm{H} 2 \mathrm{~B}$ dimers (Luger 2001). Specific proteins called histone chaperones exist to bind and stabilize histones and to control their interactions for the assembly or disassembly of nucleosomes (Gurard-Levin et al. 2014). These proteins can also alter the composition of nucleosomes by being involved in the replacement of core histones with histone variants which differ in the protein sequence and can affect both histone-DNA and histone-histone interactions to specifically change nucleosome structure and dynamics (Talbert and Henikoff 2010). In addition, some histone chaperones contribute to the removal of $\mathrm{H} 2 \mathrm{~A} / \mathrm{H} 2 \mathrm{~B}$ dimers during transcription, such as the facilitates chromatin transcription (FACT) complex (Reinberg and Sims 2006). Therefore, a reorganization of nucleosomes into substructures seems plausible, even crucial, in the context of chromatin dynamics for regulating DNA-templated processes. Thus, investigating subnucleosomal particles can provide further insight into the structure and dynamics of full nucleosomes.

As a whole, nucleosomes have been found to undergo a conformational transition upon positive torsional stress by changing their 'chirality' from a left-handed to a righthanded DNA-wrapping into a reversed nucleosomal structure called reversome (Bancaud et al. 2007). Another nucleosome conformation, termed the split nucleosome, was observed in the form of partial splitting of the $\mathrm{H} 2 \mathrm{~A} / \mathrm{H} 2 \mathrm{~B}$ dimers from the $(\mathrm{H} 3-\mathrm{H} 4)_{2}$ tetramer while remaining bound to the DNA during salt-induced disassembly, with an eventual stepwise release of the $\mathrm{H} 2 \mathrm{~A} / \mathrm{H} 2 \mathrm{~B}$ dimers and the $(\mathrm{H} 3-\mathrm{H} 4)_{2}$ tetramer (Bohm et al. 
2011). Also, passage of the transcription enzyme RNAP II through the nucleosome was found to produce a hexameric subcomplex missing one $\mathrm{H} 2 \mathrm{~A} / \mathrm{H} 2 \mathrm{~B}$ dimer, termed a hexasome (Bintu et al. 2011; Kireeva et al. 2002). The subnucleosomal structure that only contains the $(\mathrm{H} 3-\mathrm{H} 4)_{2}$ tetramer is called a tetrasome (Alilat et al. 1999). Tetrasomes were then also found to have the additional, remarkable feature of intrinsically switching between a left-handed and a right-handed 'chirality'. Thus, tetrasomes constitute an important subnucleosomal structure to study.

The nucleosome assembly protein 1 (NAP1)-mediated assembly of full nucleosomes or tetrasomes was recently investigated in real-time using freely-orbiting magnetic tweezers (FOMT) and electro-magnetic torque tweezers (eMTT) (Vlijm et al. 2015a). These novel MT techniques enable the study of the dynamics and the impact of small, and well-controlled torques on biomolecules (Janssen et al. 2012; Lipfert et al. 2011). In this study, a bare double-stranded DNA (dsDNA) molecule was tethered between the glass coverslip and a magnetic bead that was trapped by a permanent magnet of cylindrical form, with the bead allowed to freely rotate on a circular trajectory (Fig. 6a). Upon injection of the histones with NAP1, nucleosome assembly occurred instantaneously in steps that were reflected both in the tether's extension as well as the rotation angle, corresponding to the tether's twist and writhe (linking number). Nucleosome formation was also achieved by first assembling tetrasomes followed by the incorporation of the $\mathrm{H} 2 \mathrm{~A} / \mathrm{H} 2 \mathrm{~B}$ dimers. Interaction with the DNA was not observed for $\mathrm{H} 2 \mathrm{~A} / \mathrm{H} 2 \mathrm{~B}$ dimers, whereas they readily bound to previously formed tetrasomes. This observation again confirms the necessity of tetramer binding before the additional incorporation of $\mathrm{H} 2 \mathrm{~A} / \mathrm{H} 2 \mathrm{~B}$ dimers. $(\mathrm{H} 3-\mathrm{H} 4)_{2}$ tetramers assembled instantaneously onto the DNA and remained stably bound for long times, indicating that tetrasomes are viable nucleosomal substructures (Fig. 6b). Remarkably, tetrasomes were further found to spontaneously switch their 'chirality' between a preferred left-handed and a less frequently occurring right-handed DNA wrapping, which is referred to as 'handedness flipping' (Fig. 6c). These structural dynamics may explain the significantly delayed accumulation of torque in the DNA tether containing tetrasomes in torque measurements (Fig. 6d, e). The conversion of tetrasomes from one chirality state to the other by applying weak torques was suggested as the underlying mechanism for this phenomenon. In contrast, this sort of structural dynamics and behavior was not observed for nucleosomes. On the whole, this study provides new insights into the structural dynamics of nucleosomes in the context of substructures, suggesting a potential mechanism to regulate supercoiling during DNA-templated processes by absorbing the generated torque. Very similar results were obtained in a more recent follow-up study with the histone variant H3.3 (Vlijm et al. 2015b). Comparable a

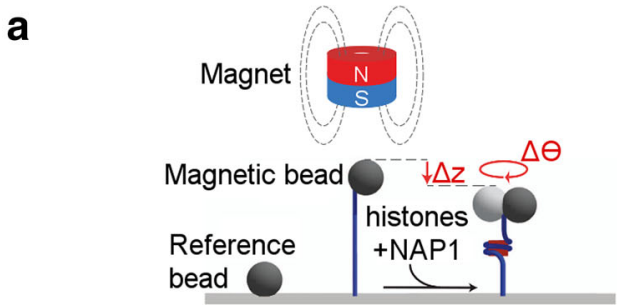

b
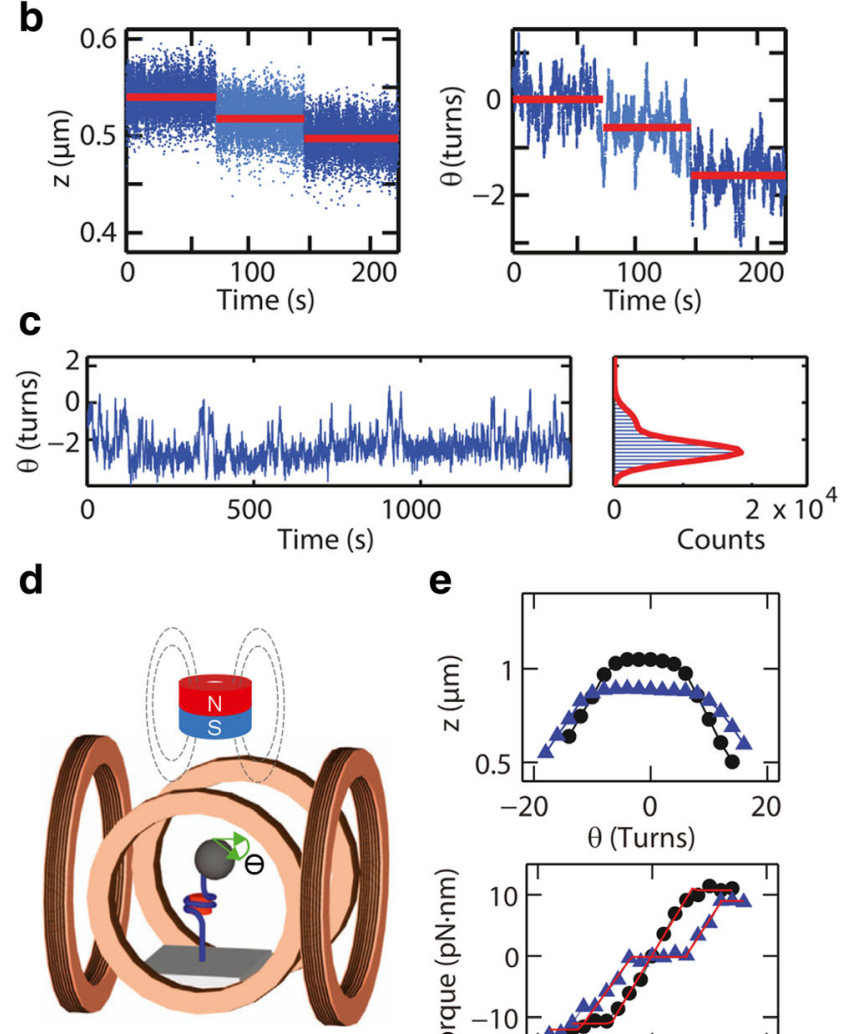

e
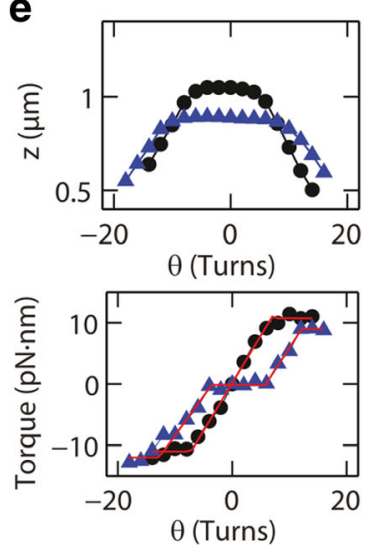

Fig. 6 Real-time assembly and structural dynamics of tetrasomes. a A DNA molecule is tethered between a glass coverslip and a magnetic bead trapped by a cylindrical magnet, thereby allowing its free rotation. The injection of histones together with nucleosome assembly protein 1 $(N A P 1)$ yields histone assembly reflected by a decrease in the tether's extension $(z$, in $\mu \mathrm{m})$ and the rotation angle $(\theta$, in turns), which is related to the tether's twist and writhe (linking number). b The extension and angle time traces show instantaneous changes in a stepwise manner upon tetrasome assembly. c The angle time traces of assembled tetrasomes reveal frequent transitions between two distinct linking numbers corresponding to a structural change in their handedness. d Two pairs of Helmholtz-coils are used to generate a horizontal magnetic field that is rotated by alternating the applied current to generate precisely controlled torques. e The rotation-extension traces of DNA molecules containing tetrasomes (blue triangles) show smaller extensions and broadening compared to the traces obtained with bare DNA (black circles), indicating assembled tethers and torque absorption, respectively. Torque absorption is verified by the rotation-torque curves revealing an additional plateau for small torques (in $\mathrm{pN} \cdot \mathrm{nm}$ ) applied to tetrasomes (blue triangles) that is absent with bare DNA (black circles). All panels $(\mathbf{a}-\mathbf{e})$ are figures reprinted or adapted from Vlijm et al. (2015a), Copyright (2015), with permission from Elsevier. This figure is not included in the present article's Creative Commons license 
details of the nucleosome assembly and the structural dynamics of tetrasomes containing the histone variant $\mathrm{H} 3.3$ indicate that the incorporation of this variant histone, which in the cell occurs upon histone loss in processes such as transcription, does not give rise to changes in nucleosomal structure and dynamics, but rather may affect other processes such as the recruitment of specific histone chaperones or remodelers.

The dynamics of (sub)nucleosomal structures were also investigated using high-speed-AFM (HS-AFM) (Katan et al. 2015). This novel technique enables the visualization of the structure and dynamics of biomolecules at acquisition rates of up to $10 \mathrm{~Hz}$ or higher (Ando et al. 2013). The histoneDNA complexes were either reconstituted by salt dialysis or assembled using NAP1 and deposited onto a mica substrate for incubation prior to imaging in liquid. Nucleosomes were found to spontaneously disassemble in a fast process on a timescale of $1 \mathrm{~s}$, while tetrasomes underwent several different dynamic changes, such as sliding, hopping between two stable positions involving a change in the 'handedness' of the DNA-wrapping and disassembly with the concomitant formation of a DNAloop that remains stable for minutes. In addition to illustrating the suitability of HS-AFM for probing DNA-protein interactions, this study reveals the highly dynamic nature of (sub)nucleosomal structures which may add an additional layer of flexibility in the accommodation and control of processes such as transcription, replication and repair.

Overall, all nucleosomal (sub)structures and their properties carry significant biological potential in the context of gene regulation during essential cellular processes. There are still many questions left to be answered on their function which could also be explained in the context of other nucleosomerelated mechanisms. Single-molecule techniques are a promising tool to advance the research on this topic by the development and application of more complex assays, as described in the next section.

\section{The nucleosome as a barrier}

As mentioned above, nucleosomes can act as dynamic mechanical barriers to related DNA-binding proteins and DNAprocessing enzymes. Many different processes that influence nucleosome structure, such as intrinsic dynamics and remodeling involving histone chaperones, chromatin remodeling enzymes as well as post-translational modifications, possibly facilitate the overcoming of the barrier. However, the exact mechanisms underlying genome processing through nucleosomes that reveal the fate of colliding enzymes and histones still remain unclear. As the first step of gene expression and one of the crucial processes to maintain cell viability and function, transcription has become a topic of great interest in the context of nucleosome research at the single-molecule level as well (Dangkulwanich et al. 2014; Teves et al. 2014).
Mimicking genome processing by unzipping dsDNA molecules containing single nucleosomes using OT revealed the locations and features of histone-DNA interactions at $\sim 1$-bp resolution (Hall et al. 2009). The 5-bp periodicity of these strong interactions within three broad regions indicates that nucleosomes actually represent a considerable energy barrier to DNA-processing enzymes. This conclusion was further confirmed by several direct studies of transcription through nucleosomes using purified RNAP II in different assays based on the common single-molecule techniques (Bintu et al. 2011, 2012; Hodges et al. 2009; Jin et al. 2010). Nucleosomes were found to have a significant effect on the dynamics of RNAP II by locally increasing the density and duration of its pausing, as well as by decreasing their actual (pause-free) velocity (Hodges et al. 2009). The authors concluded that the changes in polymerase dynamics are governed by fluctuations in nucleosome unwrapping, which would either deny or give the polymerase access to nucleosomal DNA in the closed or open nucleosome conformation, respectively. In addition to increasing the pause density and duration, nucleosomes were also observed to induce backtracking of polymerases. A successive RNAP was, however, found to release the preceding polymerase from backtracking to restart and even continue with elongation at a higher rate (Jin et al. 2010). This finding suggests that multiple RNAP II enzymes could cooperatively increase transcription efficiency. The nucleosomal barrier to transcription was further shown to be highly controlled by specific structural elements of the nucleosome (Bintu et al. 2012). Elimination of the histone tails and destabilization of specific histone-DNA interactions enabled transcription to overcome the nucleosomal barrier more easily. The greater efficiency of transcription observed for weakened histone-DNA interactions shows their essential role in nucleosome stability, while, alternatively, the histone tails could have a significant function in the recruitment and mode of action of specific remodelers. Some details of nucleosomal fate during transcription were revealed by simultaneous imaging of RNAP and nucleosomes at different stages of transcription using AFM (Bintu et al. 2011). While some nucleosomes did not change their position upon transcription, others were found upstream of their initial location, which was explained by a DNA-looping mechanism for histone transfer. In addition, some of the transcribed nucleosomes showed a smaller size depending on the elongation rate, which was ascribed to the loss of one $\mathrm{H} 2 \mathrm{~A} / \mathrm{H} 2 \mathrm{~B}$ dimer during transcription resulting in the formation of a hexamer. These studies have convincingly illustrated how transcription through nucleosomes both requires and causes structural changes that may occur by RNAPmediated changes in supercoiling and/or the action of the accessory factors, such as histone chaperones, chromatin remodelers and other transcription factors.

Single-molecule research on the transcription of nucleosome substrates is now moving towards more complex systems involving additional factors. The effect of nucleosomes 
on the binding and dissociation of transcription factors (TF) was recently studied using fluorescence microscopy, including smFRET (Luo et al. 2014). In this study, dsDNA molecules containing a binding site for either the TF LexA or the TF Gal4 were used to reconstitute nucleosomes which remained intact or were trapped in an open conformation upon TF binding. Monitoring the FRET efficiency allowed the TF binding and dissociation rates to be determined. Nucleosomes were not only found to decrease the binding rate of the TFs by $\sim 500$-fold, but also to significantly increase their dissociation rate by $\sim 1000$-fold compared to bare dsDNA molecules. These results show that nucleosomes regulate TF access to DNA and propose a possible mechanism for facilitating TF exchange. This regulatory function of the nucleosome may also apply to other DNA-binding proteins.

In another study, the effect of two general transcription elongation factors, TFIIS and TFIIF, on the transcription process of both bare and nucleosomal DNA was investigated using OT (Ishibashi et al. 2014). The specific assay comprised a dsDNA molecule directly attached to one bead at its downstream end and tethered to another bead through an RNAP II complex (Fig. 7a). Depending on the orientation of the DNA molecule, either an opposing or an assisting force could be applied to the RNAP, allowing for complementary insights into the transcription dynamics. Both TFs were added independently or together and were not found to significantly influence the actual (pause-free) elongation rate. However, the TFs did affect the pausing dynamics of the RNAP in a distinct manner (Fig. 7b). TFIIS reduced the pause duration, while TFIIF decreased both the pause density and duration depending on the force. The same effects also enhanced the progression of RNAP through the nucleosomal barrier (Fig. 7c). These single-molecule experiments thus helped to pin down the details on the dynamics and the biophysical mechanisms by which TFIIS and TFIIF enact their known positive effects on RNAP II elongation efficiency.

In a very recent study, the effects of nucleosome remodelling by either yeast SWI/SNF or ISW1a on the bound TF Gal4DBD and vice versa were investigated using the abovementioned OT-based unzipping technique ( $\mathrm{Li}$ et al. 2015). In this assay, the single-stranded DNA (ssDNA) segments of an already partially unzipped dsDNA molecule containing a single nucleosome and a bound TF were tethered between the glass coverslip and a microsphere. The molecules were further unzipped by moving the glass coverslip away from the bead, and the strengths and locations of the nucleosome and the bound TF were simultaneously reflected as peaks in the force-extension traces at $\sim 1$ bp resolution. Unzipping molecules after finalized remodeling reactions with either remodeler revealed that a TF represents a considerable barrier to remodeling by ISW1a for nucleosome repositioning. However, the SWI/SNF remodeler could generate nucleosome sliding accompanied by removal of the TF without a significant effect on nucleosome positioning. Interestingly, the nucleosomes were located in opposite directions after remodeling, indicating that both remodelers have distinct roles in nucleosome positioning in the presence of TFs. This study demonstrates a potential mechanism for the regulation of nucleosome remodeling by TFs and vice versa, which could further have a major role in the regulation of transcription in general.

In summary, the latest advances of in vitro single-molecule research on transcription involving nucleosomes represent a a



b

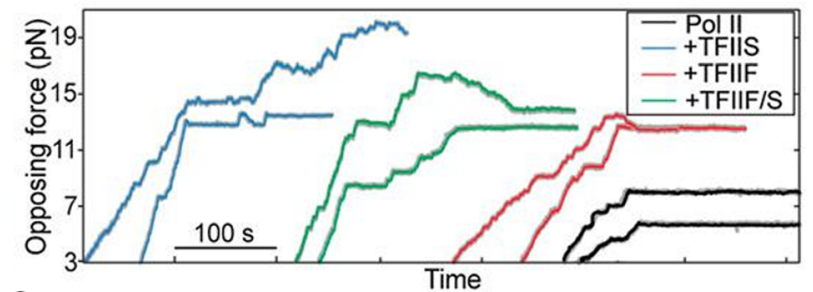

C

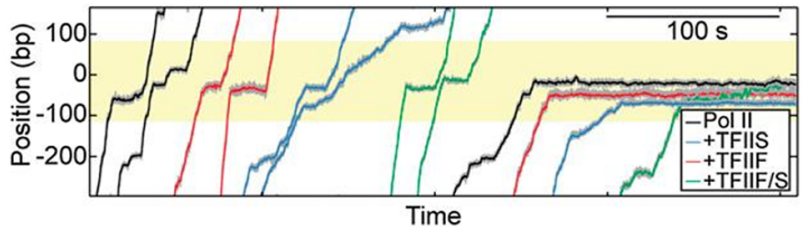

Fig. 7 Real-time dynamics of transcription enzyme RNA polymerase II (RNAP II) elongation activity in the presence of transcription elongation factors (TFs). a A DNA molecule is attached to an anti-digoxigenin (AD)-coated bead at its downstream end and tethered to another streptavidin (SA)-coated bead through an RNAP II complex with two optical traps. Two different TFs (TFIIS, blue; TFIIF, red) are injected to investigate their effect on transcription dynamics. b Time traces of the opposing force (in pN) to the transcribing RNAP II complex reveal facilitated elongation by the TFs individually (TFIIS, blue; TFIIF, red) or together (TFIIF/TFIIS, green) compared to RNAP II alone (black). c Time traces of the RNAP II position (in bp) along a DNA molecule containing an initially well-positioned single nucleosome (yellow-shaded band) show stimulation of RNAP II processivity (black) through nucleosomes by the TFs individually (TFIIS, blue; TFIIF, red) or together $(T F I I F / S$, green $)$. All panels $(\mathbf{a}-\mathbf{c})$ are figures reprinted with minor changes from Ishibashi et al. (2014), with permission from PNAS. This figure is not included in the present article's Creative Commons license 


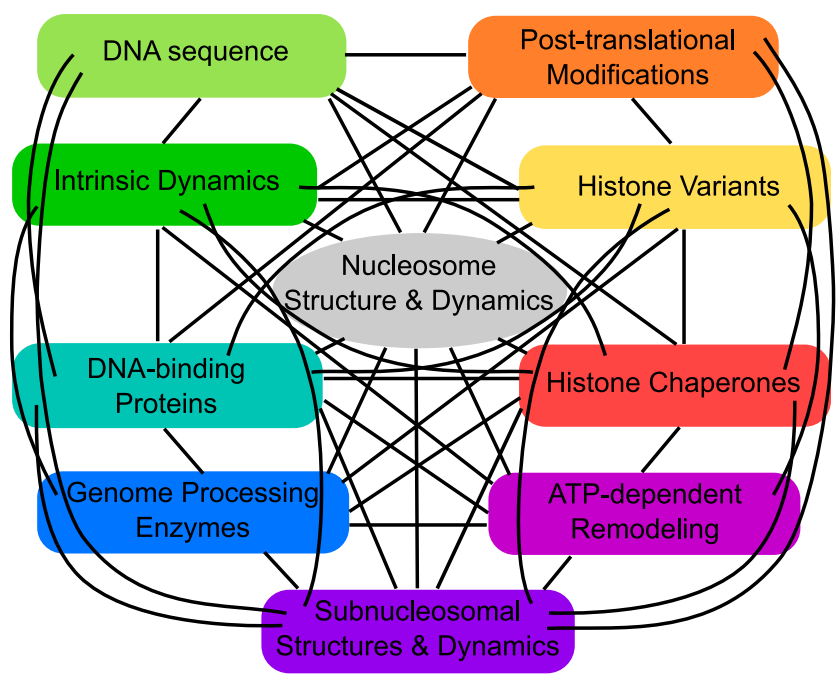

Fig. 8 Overview of mechanisms influencing nucleosome structure and dynamics. Nucleosomes are intrinsically dynamic (dark green) entities, and their structure and dynamics are additionally affected by many different mechanisms, such as sequence and chemical properties of the underlying DNA (light green), post-translational modifications of histones (orange), the incorporation of histone variants (yellow), the interactions with histone chaperones (red), ATP-dependent remodelling (magenta), the reorganization in subnucleosomal substructures (violet) and non-specifc external factors like genome processing machineries (dark blue) and DNA-binding proteins (blue/green). All these mechanisms are coherent and act in a concerted manner (black lines)

successful next step towards understanding more details of this complex process. The combination of multiple proteins and mechanisms in a crowded environment seems a promising approach to elucidate additional details of this complex fundamental process. The further development of this kind of assays is expected to also finally explain the still striking feature of transcription through chromatin in vivo occurring at similar rates to in vitro transcription of bare DNA (Izban and Luse 1992).

\section{Conclusions and future perspectives}

As the basic packaging unit of chromatin, the nucleosome represents a fundamental DNA-protein complex whose study is required for understanding the organization and regulation of the genome during essential cellular processes. In this context, nucleosomes are not static, but highly ordered and dynamic entities. Their structure and dynamics are continuously altered by different mechanisms involving spontaneous conformational changes, the properties of the underlying DNA, post-translational modifications of the histones, ATPdependent remodelers, external forces and torques, the incorporation of histone variants, and interactions with histone chaperones and other related proteins.

In this review, we have introduced the most commonly used single-molecule techniques in nucleosome research and presented recent insights they have provided into nucleosome structure, function and dynamics. Studies of individual nucleosomes in a time-resolved manner have revealed transient conformational states on fast timescales, such as breathing and possibly gaping. The structural and chemical properties of the nucleosomal DNA are suggested to influence nucleosome structure and dynamics. Post-translational modifications of histones have been found to significantly affect the intrinsic dynamics and the stability of nucleosomes by altering DNA-histone or histone-histone interactions. More assays have been developed and extended by using other nucleosome-related components, such as histone chaperones and remodeling enzymes, to study their underlying molecular mechanisms and impact on nucleosome (dis)assembly, architecture and dynamics. Various subnucleosomal structures have been identified both in vivo and in vitro and are believed to play an important role in the regulation of the genome during nuclear processes. Some great insights into the function and fate of the nucleosome as a barrier during DNA-templated processes have been provided by the incorporation of genome processing machineries. Single-molecule studies in the context of histone variants and replication could provide more insights into (sub)nucleosomal structure and dynamics and possibly reveal new mechanisms to complement current knowledge.

However, as several different mechanisms involving various proteins simultaneously act on nucleosomes in a concerted manner, many questions remain to be answered regarding the details of their interplay (Fig. 8). A powerful approach seems to be the combination of fluorescence and force spectroscopy techniques, which has already been used to probe various DNA-protein interactions (Cordova et al. 2014). Further advances in microscopy and sample preparation techniques at the single-molecule level will enable measurements in crowded conditions involving several different components and mechanisms. This will allow the recapitulation of actual biological processes in an environment that approaches in vivo conditions. In the vast majority of single-molecule methods for studying nucleosomes, specifically designed and immobilized DNA constructs containing strong nucleosome-positioning sequences, purified, engineered or labeled proteins and non-physiological buffers are used. This allows for a better control of the biological system, but in the end does not reflect the native situation. On the other hand, in vivo single-molecule studies that are mainly based on fluorescence microscopy have been very challenging due to specific treatments of live cells and in particular the low spatial resolution. However, recent developments in live cell microscopy towards super-resolution microscopy with highly improved spatial resolution hold great promise for studies of chromatin structure and dynamics in vivo (Lakadamyali and Cosma 2015). Collectively, the complementary insights from different techniques and assays will allow researchers to put individual parts of the huge puzzle on chromatin structure and dynamics together to advance our understanding of one of the fundamental aspects of life. 
Acknowledgements The authors thank Dr. Belen Solano, Dr. Aysen L. Erdem and Artur Kaczmarczyk for their critical reading of and valuable comments on the manuscript. The authors further acknowledge the many efforts in the field of chromatin research and regret that it was not possible to include more high-quality studies due to limitations in space.

\section{Compliance with ethical standards}

Funding Funding for this work was provided by the European Research Council (ERC) via a Consolidator Grant DynGenome (No:312221) to N.H.D and the Austrian Science Fund (FWF) [START Y275-B12] to A.L.

Conflict of interest Orkide Ordu declares that she does not have any conflicts of interest. Alexandra Lusser declares that she does not have any conflicts of interest. Nynke H. Dekker declares that she does not have any conflicts of interest.

Ethical approval This article does not contain any studies with human participants or animals performed by any of the authors.

Open Access This article is licensed under a Creative Commons Attribution 4.0 International License, which permits use, duplication, adaptation, distribution and reproduction in any medium or format, as long as you give appropriate credit to the original author(s) and the source, provide a link to the Creative Commons license and indicate if changes were made.

The images or other third party material in this article are included in the article's Creative Commons license, unless indicated otherwise in the credit line; if such material is not included in the article's Creative Commons license and the respective action is not permitted by statutory regulation, users will need to obtain permission from the license holder to duplicate, adapt or reproduce the material.

To view a copy of this license, visit http://creativecommons. org/licenses/by/4.0/

\section{References}

Alilat M, Sivolob A, Revet B, Prunell A (1999) Nucleosome dynamics IV. Protein and DNA contributions in the chiral transition of the tetrasome, the histone $(\mathrm{H} 3-\mathrm{H} 4)(2)$ tetramer-DNA particle. J Mol Biol 291:815-841. doi:10.1006/jmbi.1999.2988

Allen MJ, Dong XF, Oneill TE, Yau P, Kowalczykowski SC, Gatewood J, Balhorn R, Bradbury EM (1993) Atomic-force microscope measurements of nucleosome cores assembled along defined DNA-sequences. Biochemistry 32:8390-8396. doi:10.1021/bi00084a002

Amblard F, Yurke B, Pargellis A, Leibler S (1996) A magnetic manipulator for studying local rheology and micromechanical properties of biological systems. Rev Sci Instrum 67:818-827. doi:10.1063 $/ 1.1146816$

Ando T, Uchihashi T, Kodera N (2013) High-Speed AFM and applications to biomolecular systems. Annu Rev Biophys 42:393-414. doi:10.1146/annurev-biophys-083012-130324

Andrews AJ, Luger K (2011) Nucleosome structure(s) and stability: variations on a theme. Annu Rev Biophys 40:99-117. doi:10.1146 /annurev-biophys-042910-155329

Arents G, Burlingame RW, Wang BC, Love WE, Moudrianakis EN (1991) The nucleosomal core histone octamer at $3.1 \AA$ resolution: tripartite protein assembly and a left-handed superhelix. Proc Natl Acad Sci USA 88:10148-10152

Arnaudo AM, Garcia BA (2013) Proteomic characterization of novel histone post-translational modifications. Epigenetics Chromatin 6: 7. doi:10.1186/1756-8935-6-24
Ashkin A (1992) Forces of a single-beam gradient laser trap on a dielectric sphere in the ray optics regime. Biophys J 61:569-582

Ashkin A, Dziedzic JM, Bjorkholm JE, Chu S (1986) Observation of a single-beam gradient force optical trap for dielectric particles. Opt Lett 11:288-290. doi:10.1364/ol.11.000288

Axelrod D (1981) Cell-substrate contacts illuminated by total internal reflection fluorescence. J Cell Biol 89:141-145. doi:10.1083 jjcb.89.1.141

Axelrod D (2001) Total internal reflection fluorescence microscopy in cell biology. Traffic 2:764-774. doi:10.1034/j.16000854.2001.21104.x

Bancaud A, Wagner G, Cond e Silva N, Lavelle C, Wong H, Mozziconacci J, Barbi M, Sivolob A, Le Cam E, Mouawad L, Viovy JL, Victor JM, Prunell A (2007) Nucleosome chiral transition under positive torsional stress in single chromatin fibers. Mol Cell 27:135-147. doi:10.1016/j.molcel.2007.05.037

Bennink ML, Leuba SH, Leno GH, Zlatanova J, de Grooth BG, Greve J (2001) Unfolding individual nucleosomes by stretching single chromatin fibers with optical tweezers. Nat Struct Biol 8:606-610. doi:10.1038/89646

Berlier JE, Rothe A, Buller G, Bradford J, Gray DR, Filanoski BJ, Telford WG, Yue S, Liu JX, Cheung CY, Chang W, Hirsch JD, Beechem JM, Haugland RP, Haugland RP (2003) Quantitative comparison of long-wavelength Alexa Fluor dyes to Cy dyes: fluorescence of the dyes and their bioconjugates. J Histochem Cytochem 51:1699-1712

Binnig G, Quate CF, Gerber C (1986) Atomic force microscope. Phys Rev Lett 56:930-933. doi:10.1103/PhysRevLett.56.930

Bintu L, Kopaczynska M, Hodges C, Lubkowska L, Kashlev M, Bustamante C (2011) The elongation rate of RNA polymerase determines the fate of transcribed nucleosomes. Nat Struct Mol Biol 18:1394-1399. doi:10.1038/nsmb.2164

Bintu L, Ishibashi T, Dangkulwanich M, Wu YY, Lubkowska L, Kashlev M, Bustamante C (2012) Nucleosomal elements that control the topography of the barrier to transcription. Cell 151:738-749. doi:10.1016/j.cell.2012.10.009

Blosser TR, Yang JG, Stone MD, Narlikar GJ, Zhuang X (2009) Dynamics of nucleosome remodelling by individual ACF complexes. Nature 462:1022-1027. doi:10.1038/nature08627

Bohm V, Hieb AR, Andrews AJ, Gansen A, Rocker A, Toth K, Luger K, Langowski J (2011) Nucleosome accessibility governed by the dimer/tetramer interface. Nucleic Acids Res 39:3093-3102. doi: $10.1093 / \mathrm{nar} / \mathrm{gkq} 1279$

Bowman GD, Poirier MG (2015) Post-translational modifications of histones that influence nucleosome dynamics. Chem Rev 115:2274 2295. doi:10.1021/cr500350x

Brehove M, Wang T, North J, Luo Y, Dreher SJ, Shimko JC, Ottesen JJ, Luger K, Poirier MG (2015) Histone core phosphorylation regulates DNA accessibility. J Biol Chem 290:22612-22621. doi:10.1074 jjbc.M115.661363

Brower-Toland B, Wacker DA, Fulbright RM, Lis JT, Kraus WL, Wang MD (2005) Specific contributions of histone tails and their acetylation to the mechanical stability of nucleosomes. J Mol Biol 346: 135-146. doi:10.1016/j.jmb.2004.11.056

Buning R, van Noort J (2010) Single-pair FRET experiments on nucleosome conformational dynamics. Biochimie 92:1729-1740. doi:10.1016/j.biochi.2010.08.010

Butt HJ, Cappella B, Kappl M (2005) Force measurements with the atomic force microscope: technique, interpretation and applications. Surf Sci Rep 59:1-152. doi:10.1016/j.surfrep.2005.08.003

Chien FT, van Noort J (2009) 10 years of tension on chromatin: results from single molecule force spectroscopy. Curr Pharm Biotechnol 10:474-485

Choy JS, Lee TH (2012) Structural dynamics of nucleosomes at singlemolecule resolution. Trends Biochem Sci 37:425-435. doi:10.1016 j.tibs.2012.06.006 
Clapier CR, Cairns BR (2009) The biology of chromatin remodeling complexes. Annu Rev Biochem 78:273-304. doi:10.1146/annurev. biochem.77.062706.153223

Cordova JC, Das DK, Manning HW, Lang MJ (2014) Combining singlemolecule manipulation and single-molecule detection. Curr Opin Struct Biol 28:142-148. doi:10.1016/j.sbi.2014.08.010

Crick FHC, Hughes AFW (1950) The physical properties of cytoplasm: a study by means of the magnetic particle method. Part I. Experimental. Exp Cell Res 1:37-80. doi:10.1016/0014-4827 (50)90048-6

Cui Y, Bustamante C (2000) Pulling a single chromatin fiber reveals the forces that maintain its higher-order structure. Proc Natl Acad Sci USA 97:127-132. doi:10.1073/pnas.97.1.127

Dalal Y, Wang H, Lindsay S, Henikoff S (2007) Tetrameric structure of centromeric nucleosomes in interphase Drosophila cells. PLoS Biol 5:1798-1809. doi:10.1371/journal.pbio.0050218

Dangkulwanich M, Ishibashi T, Bintu L, Bustamante C (2014) Molecular mechanisms of transcription through single-molecule experiments. Chem Rev 114:3203-3223. doi:10.1021/cr400730x

Davey CA, Sargent DF, Luger K, Maeder AW, Richmond TJ (2002) Solvent mediated interactions in the structure of the nucleosome core particle at $1.9 \AA$ resolution. J Mol Biol 319:1097-1113. doi:10.1016/s0022-2836(02)00386-8

Dimitriadis EK, Weber C, Gill RK, Diekmann S, Dalal Y (2010) Tetrameric organization of vertebrate centromeric nucleosomes. Proc Natl Acad Sci USA 107:20317-20322. doi:10.1073 /pnas. 1009563107

Dulin D, Lipfert J, Moolman MC, Dekker NH (2013) Studying genomic processes at the single-molecule level: introducing the tools and applications. Nat Rev Genet 14:9-22. doi:10.1038/nrg3316

Duzdevich D, Redding S, Greene EC (2014) DNA dynamics and singlemolecule biology. Chem Rev 114:3072-3086. doi:10.1021 /cr4004117

Eickbush TH, Moudrianakis EN (1978) Histone core complex-octamer assembled by two sets of protein-protein interactions. Biochemistry 17:4955-4964. doi:10.1021/bi00616a016

Eigen M, Rigler R (1994) Sorting single molecules - application to diagnostics and evolutionary biotechnology. Proc Natl Acad Sci USA 91:5740-5747. doi:10.1073/pnas.91.13.5740

Elbel T, Langowski J (2015) The effect of DNA supercoiling on nucleosome structure and stability. J Phys Condens Matter 27:064105. doi:10.1088/0953-8984/27/6/064105

Elson EL, Magde D (1974) Fluorescence correlation spectroscopy. I. Conceptual basis and theory. Biopolymers 13:1-27. doi:10.1002 /bip.1974.360130102

Florin EL, Moy VT, Gaub HE (1994) Adhesion forces between individual ligand-receptor pairs. Science 264:415-417. doi:10.1126 /science. 8153628

Förster T (1948) Intermolecular energy migration and fluorescence [Zwischenmolekulare Energiewanderung und Fluoreszenz]. Ann Phys 2:55-75

Funatsu T, Harada Y, Tokunaga M, Saito K, Yanagida T (1995) Imaging of single fluorescent molecules and individual ATP turnovers by single myosin molecules in aqueous solution. Nature 374:555559. doi: $10.1038 / 374555 \mathrm{a} 0$

Gansen A, Valeri A, Hauger F, Felekyan S, Kalinin S, Toth K, Langowski J, Seidel CA (2009) Nucleosome disassembly intermediates characterized by single-molecule FRET. Proc Natl Acad Sci USA 106: 15308-15313. doi:10.1073/pnas.0903005106

Gemmen GJ, Sim R, Haushalter KA, Ke PC, Kadonaga JT, Smith DE (2005) Forced unraveling of nucleosomes assembled on heterogeneous DNA using core, histones, NAP-1, and ACF. J Mol Biol 351: 89-99. doi:10.1016/j.jmb.2005.05.058

Giepmans BNG, Adams SR, Ellisman MH, Tsien RY (2006) ReviewThe fluorescent toolbox for assessing protein location and function. Science 312:217-224. doi:10.1126/science.1124618
Gurard-Levin ZA, Quivy JP, Almouzni G (2014) Histone chaperones: assisting histone traffic and nucleosome dynamics. Annu Rev Biochem 83:487-517. doi:10.1146/annurev-biochem-060713035536

Ha T, Tinnefeld P (2012) Photophysics of fluorescent probes for singlemolecule biophysics and super-resolution imaging. Ann Rev Phys Chem 63:595-617. doi:10.1146/annurev-physchem-032210103340

Ha T, Enderle T, Ogletree DF, Chemla DS, Selvin PR, Weiss S (1996) Probing the interaction between two single molecules: fluorescence resonance energy transfer between a single donor and a single acceptor. Proc Natl Acad Sci USA 93:6264-6268. doi:10.1073 /pnas.93.13.6264

Hall MA, Shundrovsky A, Bai L, Fulbright RM, Lis JT, Wang MD (2009) High-resolution dynamic mapping of histone-DNA interactions in a nucleosome. Nat Struct Mol Biol 16:124-129. doi: $10.1038 / \mathrm{nsmb} .1526$

Hansma HG, Hoh JH (1994) Biomolecular imaging with the atomic-force microscope. Annu Rev Biophys Biomol Struct 23:115-139. doi:10.1146/annurev.bb.23.060194.000555

Hell SW (2009) Microscopy and its focal switch. Nat Methods 6:24-32. doi:10.1038/nmeth.1291

Heller I, Hoekstra TP, King GA, Peterman EJG, Wuite GJL (2014) Optical tweezers analysis of DNA-protein complexes. Chem Rev 114:3087-3119. doi:10.1021/cr4003006

Hodges C, Bintu L, Lubkowska L, Kashlev M, Bustamante C (2009) Nucleosomal fluctuations govern the transcription dynamics of RNA polymerase II. Science 325:626-628. doi:10.1126 /science. 1172926

Hota SK, Bartholomew B (2011) Diversity of operation in ATPdependent chromatin remodelers. Biochim Biophys Acta 1809: 476-487. doi:10.1016/j.bbagrm.2011.05.007

Huang B, Bates M, Zhuang XW (2009) Super-resolution fluorescence microscopy. Annu Rev Biochem 78:993-1016. doi:10.1146 /annurev.biochem.77.061906.092014

Hwang WL, Deindl S, Harada BT, Zhuang X (2014) Histone H4 tail mediates allosteric regulation of nucleosome remodelling by linker DNA. Nature 512:213-217. doi:10.1038/nature13380

Ishibashi T, Dangkulwanich M, Coello Y, Lionberger TA, Lubkowska L, Ponticelli AS, Kashlev M, Bustamante C (2014) Transcription factors IIS and IIF enhance transcription efficiency by differentially modifying RNA polymerase pausing dynamics. Proc Natl Acad Sci USA 111:3419-3424. doi:10.1073/pnas.1401611111

Izban MG, Luse DS (1992) Factor-stimulated rna polymerase II transcribes at physiological elongation rates on naked DNA but very poorly on chromatin templates. J Biol Chem 267:13647-13655

Janssen XJA, Lipfert J, Jager T, Daudey R, Beekman J, Dekker NH (2012) Electromagnetic Torque Tweezers: a versatile approach for measurement of single-molecule twist and torque. Nano Lett 12: 3634-3639. doi:10.1021/nl301330h

Jares-Erijman EA, Jovin TM (2003) FRET imaging. Nat Biotechnol 21: 1387-1395. doi:10.1038/nbt896

Jin J, Bai L, Johnson DS, Fulbright RM, Kireeva ML, Kashlev M, Wang MD (2010) Synergistic action of RNA polymerases in overcoming the nucleosomal barrier. Nat Struct Mol Biol 17:745-752. doi:10.1038/nsmb.1798

Joo C, Balci H, Ishitsuka Y, Buranachai C, Ha T (2008) Advances in single-molecule fluorescence methods for molecular biology. Annu Rev Biochem 77:51-76. doi:10.1146/annurev. biochem.77.070606.101543

Katan AJ, Vlijm R, Lusser A, Dekker C (2015) Dynamics of nucleosomal structures measured by high-speed atomic force microscopy. Small 11:976-984. doi:10.1002/smll.201401318

Kidwell MG (2002) Transposable elements and the evolution of genome size in eukaryotes. Genetica 115:49-63. doi:10.1023 /a:1016072014259 
Killian JL, Li M, Sheinin MY, Wang MD (2012) Recent advances in single molecule studies of nucleosomes. Curr Opin Struct Biol 22: 80-87. doi:10.1016/j.sbi.2011.11.003

Kireeva ML, Walter W, Tchernajenko V, Bondarenko V, Kashlev M, Studitsky VM (2002) Nucleosome remodeling induced by RNA polymerase II: Loss of the $\mathrm{H} 2 \mathrm{~A} / \mathrm{H} 2 \mathrm{~B}$ dimer during transcription. Mol Cell 9:541-552. doi:10.1016/s1097-2765(02)00472-0

Klug A, Rhodes D, Smith J, Finch JT, Thomas JO (1980) A low resolution structure for the histone core of the nucleosome. Nature 287: 509-516

Koopmans WJA, Brehm A, Logie C, Schmidt T, van Noort J (2007) Single-pair FRET microscopy reveals mononucleosome dynamics. J Fluoresc 17:785-795. doi:10.1007/s10895-007-0218-9

Koopmans WJ, Buning R, Schmidt T, van Noort J (2009) spFRET using alternating excitation and FCS reveals progressive DNA unwrapping in nucleosomes. Biophys J 97:195-204. doi:10.1016 /j.bpj.2009.04.030

Kornberg RD (1974) Chromatin structure: a repeating unit of histones and DNA. Science 184:868-871

Kornberg RD (1977) Structure of chromatin. Annu Rev Biochem 46: 931-954. doi:10.1146/annurev.bi.46.070177.004435

Kornberg RD, Lorch YL (1999) Twenty-five years of the nucleosome, fundamental particle of the eukaryote chromosome. Cell 98:285294. doi:10.1016/s0092-8674(00)81958-3

Kruithof M, Chien FT, Routh A, Logie C, Rhodes D, van Noort J (2009) Single-molecule force spectroscopy reveals a highly compliant helical folding for the 30-nm chromatin fiber. Nat Struct Mol Biol 16: 534-540. doi:10.1038/nsmb.1590

La Porta A, Wang MD (2004) Optical Torque Wrench: angular trapping, rotation, and torque detection of quartz microparticles. Phys Rev Lett 92:190801. doi:10.1103/PhysRevLett.92.190801

Lakadamyali M, Cosma MP (2015) Advanced microscopy methods for visualizing chromatin structure. FEBS Lett 589:3023-3030. doi:10.1016/j.febslet.2015.04.012

Lavelle C, Prunell A (2007) Chromatin polymorphism and the nucleosome superfamily - a genealogy. Cell Cycle 6:2113-2119

Lavelle C, Victor JM, Zlatanova J (2010) Chromatin fiber dynamics under tension and torsion. Int J Mol Sci 11:1557-1579. doi:10.3390/ijms11041557

Lavelle C, Praly E, Bensimon D, Le Cam E, Croquette V (2011) Nucleosome-remodelling machines and other molecular motors observed at the single-molecule level. FEBS J 278:3596-3607. doi:10.1111/j.1742-4658.2011.08280.x

Leuba SH, Karymov MA, Tomschik M, Ramjit R, Smith P, Zlatanova J (2003) Assembly of single chromatin fibers depends on the tension in the DNA molecule: magnetic tweezers study. Proc Natl Acad Sci USA 100:495-500. doi:10.1073/pnas.0136890100

Li B, Carey M, Workman JL (2007) The role of chromatin during transcription. Cell 128:707-719. doi:10.1016/j.cell.2007.01.015

Li G, Levitus M, Bustamante C, Widom J (2005) Rapid spontaneous accessibility of nucleosomal DNA. Nat Struct Mol Biol 12:46-53. doi: $10.1038 / \mathrm{nsmb} 869$

Li M, Hada A, Sen P, Olufemi L, Hall MA, Smith BY, Forth S, McKnight JN, Patel A, Bowman GD, Bartholomew B, Wang MD (2015) Dynamic regulation of transcription factors by nucleosome remodeling. Elife 4. doi:10.7554/eLife.06249

Lia G, Praly E, Ferreira H, Stockdale C, Tse-Dinh YC, Dunlap D, Croquette V, Bensimon D, Owen-Hughes T (2006) Direct observation of DNA distortion by the RSC complex. Mol Cell 21:417-425. doi:10.1016/j.molcel.2005.12.013

Lipfert J, Wiggin M, Kerssemakers JWJ, Pedaci F, Dekker NH (2011) Freely orbiting magnetic tweezers to directly monitor changes in the twist of nucleic acids. Nat Commun 2:9. doi:10.1038/ncomms1450

Luger K (2001) Nucleosomes: structure and function. In: eLS. John Wiley \& Sons Ltd, Chichester. http://www.els.net [doi:10.1038 /npg.els.0001155]
Luger K, Mäder AW, Richmond RK, Sargent DF, Richmond TJ (1997) Crystal structure of the nucleosome core particle at $2.8 \AA$ resolution. Nature 389:251-260

Luger K, Dechassa ML, Tremethick DJ (2012) New insights into nucleosome and chromatin structure: an ordered state or a disordered affair? Nat Rev Mol Cell Biol 13:436-447. doi:10.1038/nrm3382

Luo Y, North JA, Rose SD, Poirier MG (2014) Nucleosomes accelerate transcription factor dissociation. Nucleic Acids Res 42:3017-3027. doi:10.1093/nar/gkt1319

Mack AH, Schlingman DJ, Ilagan RP, Regan L, Mochrie SGJ (2012) Kinetics and thermodynamics of phenotype: unwinding and rewinding the nucleosome. J Mol Biol 423:687-701. doi:10.1016/j. jmb.2012.08.021

Magde D, Webb WW, Elson E (1972) Thermodynamic fluctuations in a reacting system - measurement by fluorescence correlation spectroscopy. Phys Rev Lett 29:705. doi:10.1103/PhysRevLett.29.705

Magde D, Elson EL, Webb WW (1974) Fluorescence correlation spectroscopy. II. Experimental realization. Biopolymers 13:29-61. doi:10.1002/bip.1974.360130103

McGhee JD, Felsenfeld G (1980) Nucleosome structure. Annu Rev Biochem 49:1115-1156. doi:10.1146/annurev. bi.49.070180.005343

Merhej V, Raoult D (2012) Rhizome of life, catastrophes, sequence exchanges, gene creations, and giant viruses: how microbial genomics challenges Darwin. Front Cell Infect Microbiol 2:17. doi:10.3389 /fcimb.2012.00113

Minsky M (1988) Memoir on inventing the confocal scanning microscope. Scanning 10:128-138

Miyagi A, Ando T, Lyubchenko YL (2011) Dynamics of nucleosomes assessed with time-lapse high-speed atomic force microscopy. Biochemistry 50:7901-7908. doi:10.1021/bi200946z

Moerner WE (2007) New directions in single-molecule imaging and analysis. Proc Natl Acad Sci USA 104:12596-12602. doi:10.1073 /pnas.0610081104

Moerner WE, Fromm DP (2003) Methods of single-molecule fluorescence spectroscopy and microscopy. Rev Sci Instrum 74:35973619. doi:10.1063/1.1589587

Monico C, Capitanio M, Belcastro G, Vanzi F, Pavone FS (2013) Optical methods to study protein-DNA interactions in vitro and in living cells at the single-molecule level. Int J Mol Sci 14:3961-3992. doi:10.3390/ijms14023961

Neuman KC, Nagy A (2008) Single-molecule force spectroscopy: optical tweezers, magnetic tweezers and atomic force microscopy. Nat Methods 5:491-505. doi:10.1038/nmeth.1218

Neuman KC, Lionnet T, Allemand JF (2007) Single-molecule micromanipulation techniques. Annu Rev Mater Res 37:33-67. doi:10.1146 /annurev.matsci.37.052506.084336

Ngo TT, Ha T (2015) Nucleosomes undergo slow spontaneous gaping. Nucleic Acids Res 43:3964-3971. doi:10.1093/nar/gkv276

Ngo TT, Zhang Q, Zhou R, Yodh JG, Ha T (2015) Asymmetric unwrapping of nucleosomes under tension directed by DNA local flexibility. Cell 160:1135-1144. doi:10.1016/j.cell.2015.02.001

Nie SM, Chiu DT, Zare RN (1994) Probing individual molecules with confocal fluorescence microscopy. Science 266:1018-1021. doi: $10.1126 /$ science. 7973650

North JA, Shimko JC, Javaid S, Mooney AM, Shoffner MA, Rose SD, Bundschuh R, Fishel R, Ottesen JJ, Poirier MG (2012) Regulation of the nucleosome unwrapping rate controls DNA accessibility. Nucleic Acids Res 40:10215-10227. doi:10.1093/nar/gks 747

Olins AL, Olins DE (1974) Spheroid chromatin units ( $v$ bodies). Science 183:330-332

Oudet P, Gross-Bellard M, Chambon P (1975) Electron microscopic and biochemical evidence that chromatin structure is a repeating unit. Cell 4:281-300 
Petesch SJ, Lis JT (2012) Overcoming the nucleosome barrier during transcript elongation. Trends Genet 28:285-294. doi:10.1016/j. tig.2012.02.005

Poirier MG, Oh E, Tims HS, Widom J (2009) Dynamics and function of compact nucleosome arrays. Nat Struct Mol Biol 16:938-U959. doi: $10.1038 / \mathrm{nsmb} .1650$

Polo SE, Almouzni G (2006) Chromatin assembly: a basic recipe with various flavours. Curr Opin Genet Dev 16:104-111. doi:10.1016/j. gde.2006.02.011

Pope LH, Bennink ML, van Leijenhorst-Groener KA, Nikova D, Greve J, Marko JF (2005) Single chromatin fiber stretching reveals physically distinct populations of disassembly events. Biophys J 88:35723583. doi:10.1529/biophysj.104.053074

Reinberg D, Sims RJ (2006) De FACTo nucleosome dynamics. J Biol Chem 281:23297-23301. doi:10.1074/jbc.R600007200

Richmond TJ, Finch JT, Rushton B, Rhodes D, Klug A (1984) Structure of the nucleosome core particle at $7 \AA$ resolution. Nature 311:532-537

Rigler R, Mets U, Widengren J, Kask P (1993) Fluorescence correlation spectroscopy with high count rate and low-background - analysis of translational diffusion. Eur Biophys J Biophys 22:169-175

Schmidt T, Schutz GJ, Baumgartner W, Gruber HJ, Schindler H (1996) Imaging of single molecule diffusion. Proc Natl Acad Sci USA 93: 2926-2929. doi:10.1073/pnas.93.7.2926

Shaner NC, Steinbach PA, Tsien RY (2005) A guide to choosing fluorescent proteins. Nat Methods 2:905-909. doi:10.1038/nmeth819

Sheinin MY, Li M, Soltani M, Luger K, Wang MD (2013) Torque modulates nucleosome stability and facilitates $\mathrm{H} 2 \mathrm{~A} / \mathrm{H} 2 \mathrm{~B}$ dimer loss. Nat Commun 4:8. doi:10.1038/ncomms3579

Shlyakhtenko LS, Lushnikov AY, Lyubchenko YL (2009) Dynamics of nucleosomes revealed by time-lapse atomic force microscopy. Biochemistry 48:7842-7848. doi:10.1021/bi900977t

Shundrovsky A, Smith CL, Lis JT, Peterson CL, Wang MD (2006) Probing SWI/SNF remodeling of the nucleosome by unzipping single DNA molecules. Nat Struct Mol Biol 13:549-554. doi:10.1038/nsmb1102

Simon M, North JA, Shimko JC, Forties RA, Ferdinand MB, Manohar M, Zhang M, Fishel R, Ottesen JJ, Poirier MG (2011) Histone fold modifications control nucleosome unwrapping and disassembly. Proc Natl Acad Sci USA 108:12711-12716. doi:10.1073 /pnas. 1106264108

Sirinakis G, Clapier CR, Gao Y, Viswanathan R, Cairns BR, Zhang YL (2011) The RSC chromatin remodelling ATPase translocates DNA with high force and small step size. EMBO J 30:2364-2372. doi:10.1038/emboj.2011.141

Smith SB, Finzi L, Bustamante C (1992) Direct mechanical measurements of the elasticity of single DNA-molecules by using magnetic beads. Science 258:1122-1126. doi:10.1126/science.1439819

Smith SB, Cui YJ, Bustamante C (1996) Overstretching B-DNA: The elastic response of individual double-stranded and single-stranded DNA molecules. Science 271:795-799. doi:10.1126/science.271.5250.795

Strick TR, Allemand JF, Bensimon D, Bensimon A, Croquette V (1996) The elasticity of a single supercoiled DNA molecule. Science 271: 1835-1837. doi:10.1126/science.271.5257.1835

Strick TR, Allemand JF, Bensimon D, Croquette V (1998) Behavior of supercoiled DNA. Biophys J 74:2016-2028

Talbert PB, Henikoff S (2010) Histone variants - ancient wrap artists of the epigenome. Nat Rev Mol Cell Biol 11:264-275. doi:10.1038 /nrm2861

Teves SS, Weber CM, Henikoff S (2014) Transcribing through the nucleosome. Trends Biochem Sci 39:577-586. doi:10.1016/j. tibs.2014.10.004

Tims HS, Gurunathan K, Levitus M, Widom J (2011) Dynamics of nucleosome invasion by DNA binding proteins. J Mol Biol 411:430448. doi:10.1016/j.jmb.2011.05.044
Tinoco I, Gonzalez RL (2011) Biological mechanisms, one molecule at a time. Genes Dev 25:1205-1231. doi:10.1101/gad.2050011

Tomschik M, Zheng HC, van Holde K, Zlatanova J, Leuba SH (2005) Fast, long-range, reversible conformational fluctuations in nucleosomes revealed by single-pair fluorescence resonance energy transfer. Proc Natl Acad Sci USA 102:3278-3283. doi:10.1073 /pnas.0500189102

Tomschik M, van Holde K, Zlatanova J (2009) Nucleosome dynamics as studied by single-pair fluorescence resonance energy transfer: a reevaluation. J Fluoresc 19:53-62. doi:10.1007/s10895-008-0379-1

Trifonov EN, Nibhani R (2015) Review fifteen years of search for strong nucleosomes. Biopolymers 103:432-437. doi:10.1002/bip.22604

Vlijm R, Smitshuijzen JSJ, Lusser A, Dekker C (2012) NAP1-assisted nucleosome assembly on DNA measured in real time by singlemolecule magnetic tweezers. PLoS One 7:11. doi:10.1371/journal. pone.0046306

Vlijm R, Lee M, Lipfert J, Lusser A, Dekker C, Dekker NH (2015a) Nucleosome assembly dynamics involve spontaneous fluctuations in the handedness of tetrasomes. Cell Rep 10:216-225. doi:10.1016 j.celrep.2014.12.022

Vlijm R, Lee M, Ordu O, Boltengagen A, Lusser A, Dekker NH, Dekker $\mathrm{C}$ (2015b) Comparing the assembly and handedness dynamics of (H3.3-H4)2 tetrasomes to canonical tetrasomes. PLoS One 10: e0141267. doi:10.1371/journal.pone.0141267

Wang MD, Schnitzer MJ, Yin H, Landick R, Gelles J, Block SM (1998) Force and velocity measured for single molecules of RNA polymerase. Science 282:902-907. doi:10.1126/science.282.5390.902

Wei S, Falk SJ, Black BE, Lee TH (2015) A novel hybrid single molecule approach reveals spontaneous DNA motion in the nucleosome. Nucleic Acids Res 43:e111. doi:10.1093/nar/gkv549

Weiss S (1999) Fluorescence spectroscopy of single biomolecules. Science 283:1676-1683. doi:10.1126/science.283.5408.1676

Widom J (2001) Role of DNA sequence in nucleosome stability and dynamics. Q Rev Biophys 34:269-324

Workman JL, Kingston RE (1998) Alteration of nucleosome structure as a mechanism of transcriptional regulation. Annu Rev Biochem 67: 545-579. doi:10.1146/annurev.biochem.67.1.545

Yan J, Maresca TJ, Skoko D, Adams CD, Xiao BT, Christensen MO, Heald R, Marko JF (2007) Micromanipulation studies of chromatin fibers in Xenopus egg extracts reveal ATP-dependent chromatin assembly dynamics. Mol Biol Cell 18:464-474. doi:10.1091/mbc. E06-09-0800

Yoda K, Ando S, Morishita S, Houmura K, Hashimoto K, Takeyasu K, Okazaki T (2000) Human centromere protein A (CENP-A) can replace histone $\mathrm{H} 3$ in nucleosome reconstitution in vitro. Proc Natl Acad Sci USA 97:7266-7271. doi:10.1073/pnas.130189697

Zentner GE, Henikoff S (2013) Regulation of nucleosome dynamics by histone modifications. Nat Struct Mol Biol 20:259-266. doi: $10.1038 / \mathrm{nsmb} .2470$

Zhang YL, Smith CL, Saha A, Grill SW, Mihardja S, Smith SB, Cairns BR, Peterson CL, Bustamantel C (2006) DNA translocation and loop formation mechanism of chromatin remodeling by SWI/SNF and RSC. Mol Cell 24:559-568. doi:10.1016/j. molcel.2006.10.025

Ziemann F, Radler J, Sackmann E (1994) Local measurements of viscoelastic moduli of entangled actin networks using an oscillating magnetic bead micro-rheometer. Biophys J 66:2210-2216

Zimmer C (2007) Evolution - Jurassic genome. Science 315:1358-1359. doi:10.1126/science.315.5817.1358

Zlatanova J, Leuba SH (2003) Chromatin fibers, one-at-a-time. J Mol Biol 331:1-19. doi:10.1016/s0022-2836(03)00691-0

Zlatanova J, Bishop TC, Victor JM, Jackson V, van Holde K (2009) The nucleosome family: Dynamic and growing. Structure 17:160-171. doi:10.1016/j.str.2008.12.016 